\title{
Multiple Facets for Dynamic Information Flow
}

\author{
Thomas H. Austin Cormac Flanagan \\ taustin@ucsc.edu_cormac@ucsc.edu \\ University of California, Santa Cruz
}

\begin{abstract}
JavaScript has become a central technology of the web, but it is also the source of many security problems, including cross-site scripting attacks and malicious advertising code. Central to these problems is the fact that code from untrusted sources runs with full privileges. We implement information flow controls in Firefox to help prevent violations of data confidentiality and integrity.

Most previous information flow techniques have primarily relied on either static type systems, which are a poor fit for JavaScript, or on dynamic analyses that sometimes get stuck due to problematic implicit flows, even in situations where the target web application correctly satisfies the desired security policy.

We introduce faceted values, a new mechanism for providing information flow security in a dynamic manner that overcomes these limitations. Taking inspiration from secure multi-execution, we use faceted values to simultaneously and efficiently simulate multiple executions for different security levels, thus providing non-interference with minimal overhead, and without the reliance on the stuck executions of prior dynamic approaches.
\end{abstract}

Categories and Subject Descriptors D.3.3 [Programming Languages]: Language Constructs and Features; D.4.6 [Operating Systems]: Security and Protection-Information flow controls

General Terms Languages, Security

Keywords Information flow control, dynamic analysis, JavaScript, web security

\section{Introduction}

JavaScript has helped to usher in a new age of richly interactive web applications. Often times, developers build these sites by including JavaScript code from a number of different sources. With minimal effort, a web developer can build an impressive site by composing code from multiple sources.

Unfortunately, there are few restrictions on the included code, and it operates with the same authority as the web developer's own code. Advertising has been a particular source of malicious JavaScript. There are a wide array of security measures used to defend against these problems, but the bulk of them tend to rely on competent web developers. Given the mercurial nature of security challenges, even a conscientious web developer has difficulty keeping up with the latest trends and best practices.

Permission to make digital or hard copies of all or part of this work for personal or classroom use is granted without fee provided that copies are not made or distributed for profit or commercial advantage and that copies bear this notice and the full citation on the first page. To copy otherwise, to republish, to post on servers or to redistribute to lists, requires prior specific permission and/or a fee.

POPL'12, January 25-27, 2012, Philadelphia, PA, USA.

Copyright (C) 2012 ACM 978-1-4503-1083-3/12/01...\$5.00
Another option is to bake security controls into the browser itself. This strategy has been part of browser design since nearly the beginning, but the controls have tended to be fairly minimal.

Information flow analysis offers the promise of a systematic solution to many of these security challenges, but to date it has not achieved its potential, largely because much research on static information flow type systems is an awkward fit for dynamically typed JavaScript code. Additionally, there has been a folklore that dynamic information flow analysis is not sound in the presence of implicit flows.

This folklore is not true, however, and proposed mechanisms for dealing with implicit flows include the no-sensitive-upgrade semantics $[39,5]$ and the permissive-upgrade semantics [6]. Both semantics guarantee the key correctness property of terminationinsensitive non-interference (TINI), which states that private inputs do not influence public outputs. (Private information can influence termination, but this channel is limited to a brute force attack [1]).

Despite this correctness guarantee, neither semantics provides an ideal foundation for JavaScript security since both suffer from the same weakness: in the presence of subtle implicit flows that are hard to track, the semantics halts execution in order to avoid any (potential) information leak. Note that this fail-stop is not caused by the web application violating a security policy; instead it is a mechanism failure caused by the inability of the dynamic information flow analysis to track implicit flows. Thus, these dynamic analyses reject valid programs that conform to the security policy.

An interesting solution to these mechanism failures is to simultaneously execute two copies of the target program: a highconfidentiality $(\mathrm{H})$ process that has access to secret data, and a low-confidentiality (L) process that sees dummy default values instead of the actual secret data $[9,13]$. This multi-process execution cleanly guarantees non-interference since no information flow is permitted between the two processes, and it also avoids mechanism failures. Unfortunately, for a web page with $n$ principals (roughly, URL domains), we may require up to $2^{n}$ processes, one for each element in the powerset lattice for these principals.

In this paper, we combine the benefits of multi-process execution with the efficiency of single-process execution. The key technical novelty is the introduction of a faceted value, which is a pair of two raw values that contain low and high confidentiality information, respectively. By appropriately manipulating these faceted values, a single process can simulate the two processes ( $\mathrm{L}$ and $\mathrm{H})$ of the multi-execution approach. The primary benefit of this approach is that, for most data, the two raw values in a faceted value are identical, in which case we collapse the two simulated executions on identical data into a single execution, drastically reducing the overhead. In the presence of multiple principals and a complex security lattice, a faceted value can contain many raw values, rather than just two. In this situation, the semantics of tracking information flow is a little more complex, costing some run-time performance overhead. However, our experimental results suggest that faceted 
evaluation outperforms multi-process execution as the number of principals increases.

This paper includes a formal description of the faceted value approach to dynamic information flow analysis, and a proof that it achieves termination-insensitive non-interference. We also present a projection theorem showing that a computation over faceted values simulates $2^{n}$ non-faceted computations, one for each element in the powerset security lattice. We have implemented this mechanism inside the Firefox browser (using the Zaphod plug-in [26]) in order to validate its utility in a web browsing context. Additionally, we have used this implementation to compare the performance of faceted values against multi-process execution. Finally, we discuss declassification and how it relates to faceted values, noting this feature as an additional point of distinction with multi-process execution.

\subsection{Overview of Faceted Evaluation}

To motivate the need for faceted values in dynamic information flow, we start by considering the classic problem of implicit flows, such as those caused by a conditional assignment:

$$
\text { if ( } x \text { ) } y=\text { true }
$$

The central insight of this paper is that the correct value for $y$ after this assignment depends on the authority of the observer. For example, suppose initially that $x=$ true and $y=$ false, and that $\mathrm{x}$ is secret whereas $\mathrm{y}$ is public. Then after this assignment:

- A private observer that can read $\mathrm{x}$ should see $\mathrm{y}=$ true.

- A public observer that cannot read $\mathrm{x}$ should see $\mathrm{y}=$ false, since it should not see any influence from this conditional assignment.

Faceted values represent exactly this dual nature of $y$, which should simultaneously appear as true and false to different observers.

In more detail, a faceted value is a triple consisting of a principal $k$ and two values $V_{\mathrm{H}}$ and $V_{\mathrm{L}}$, which we write as:

$$
\left\langle k ? V_{\mathrm{H}}: V_{\mathrm{L}}\right\rangle
$$

Intuitively, this faceted value appears as $V_{\mathrm{H}}$ to private observers that can view $k$ 's private data, and as $V_{\mathrm{L}}$ to other public observers. We refer to $V_{\mathrm{H}}$ and $V_{\mathrm{L}}$ as private and public facets, respectively.

This faceted value representation naturally generalizes the traditional public and private security labels used by prior analyses. A public value $V$ is represented in our setting simply as $V$ itself, since $V$ appears the same to both public and private observers and so no facets are needed. A private value $V$ is represented as the faceted value

$$
\langle k ? V: \perp\rangle
$$

where only private observers can see $V$, and where public or unauthorized observers instead see $\perp$ (roughly meaning undefined).

Although the notions of public and private data have been well explored by earlier dynamic information flow analyses, these two security labels are insufficient to avoid stuck executions in the presence of implicit flows. As illustrated by the conditional assignment considered above, correct handling of implicit flows requires the introduction of more general notion of faceted values $\left\langle k\right.$ ? $\left.V_{\mathrm{H}}: V_{\mathrm{L}}\right\rangle$, in which the public facet $V_{\mathrm{L}}$ is a real value and not simply $\perp$. In particular, the post-assignment value for $\mathrm{y}$ is cleanly represented as the faceted value $\langle k$ ? true : false $\rangle$ that captures y's appearance to both public and private observers.

Based on this faceted value representation, this paper develops a dynamic analysis that tracks information flow in a sound manner at runtime. Our analysis is formulated as an evaluation semantics for the target program, where the semantics uses faceted values to track security and dependency information.
This evaluation semantics is designed to avoid leaking information between public and private facets. In particular, if $C[\bullet]$ is any program context, then the computation $C\left[\left\langle k ? V_{\mathrm{H}}: V_{\mathrm{L}}\right\rangle\right]$ appears to behave exactly like $C\left[V_{\mathrm{H}}\right]$ from the perspective of a private observer, and behaves exactly like $C\left[V_{\mathrm{L}}\right]$ to a public observer (under a termination-insensitive notion of equivalence). This projection property means that a single faceted computation simulates multiple non-faceted computations, one for each element in the security lattice. This projection property also enables an elegant proof of termination-insensitive non-interference, shown in Section 3.2.

Faceted values may be nested. Nested faceted values naturally arise during computations with multiple principals. For example, if $k_{1}$ and $k_{2}$ denote different principals, then the expression

$$
\left\langle k_{1} \text { ? true }: \perp\right\rangle \text { \&\& }\left\langle k_{2} \text { ? false }: \perp\right\rangle
$$

evaluates to the nested faceted value

$$
\left\langle k_{1} ?\left\langle k_{2} \text { ? false }: \perp\right\rangle: \perp\right\rangle
$$

since the result $f a l s e$ is visible only to observers authorized to see private data from both $k_{1}$ and $k_{2}$; any other observer instead sees the dummy value $\perp$.

As a second example, the expression

$$
\left\langle k_{1} ? 2: 0\right\rangle+\left\langle k_{2} ? 1: 0\right\rangle
$$

evaluates to the result

$$
\left\langle k_{1} ?\left\langle k_{2} ? 3: 2\right\rangle:\left\langle k_{2} ? 1: 0\right\rangle\right\rangle
$$

Thus, faceted values form binary trees with principals at interior nodes and raw (non-faceted) values at the leaves. The part of this faceted value tree that is actually seen by a particular observer depends on whose private data the observer can read. In particular, we define the view of an observer as the set of principals whose private data that observer can read. Thus, an observer with view $\left\{k_{1}, k_{2}\right\}$ would see the result of 3 from this addition, whereas an observer with view $\left\{k_{2}\right\}$ would see the result 1 .

When a faceted value influences the control flow, in general we may need to explore the behavior of the program under both facets ${ }^{1}$. For example, the evaluation of the conditional expression:

$$
\text { if ( }\langle k \text { ? true: false }\rangle \text { ) then } e_{1} \text { else } e_{2}
$$

evaluates both $e_{1}$ and $e_{2}$, and carefully tracks the dependency of these computations on the principal $k$. In particular, assignments performed during $e_{1}$ are visible only to views that include $k$, while assignments performed during $e_{2}$ are visible to views that exclude $k$. After the evaluations of $e_{1}$ and $e_{2}$ complete, their two results are combined into a single faceted value that is returned to the continuation of this conditional expression. That is, the execution is split only for the duration of this conditional expression, rather than for the remainder of the entire program.

\subsection{Handling Implicit Flows}

The key challenge in dynamic information flow analysis lies in handling implicit flows. To illustrate this difficulty, consider the code in the first column of Figure 1, which is adapted from an example by Fenton [16]. Here, the function $f(x)$ returns the value of its boolean argument $\mathrm{x}$, but it first attempts to "launder" this value by encoding it in the program counter.

We consider the evaluation of $f$ on the two secret arguments $\langle k$ ? false : $\perp\rangle$ and $\langle k$ ? true : $\perp\rangle$ (analogous to the more traditional false ${ }^{H}$ and true $\left.{ }^{H}\right)$ to determine if the argument in any way influences any public component of the function's result.

For the argument $\langle k ?$ false : $\perp\rangle$ shown in column 2 , the local variables $\mathrm{y}$ and $\mathrm{z}$ are initialized to true. The conditional

\footnotetext{
${ }^{1}$ The semantics is optimized to avoid such split executions where possible.
} 
Figure 1: A JavaScript Function with Implicit Flows

\begin{tabular}{|c|c|c|c|c|c|}
\hline \multirow[b]{2}{*}{ Function $f(x)$} & $\mathrm{x}=\langle k ? \mathrm{false}: \perp\rangle$ & \multicolumn{4}{|c|}{$\mathrm{x}=\langle k ?$ true $: \perp\rangle$} \\
\hline & All strategies & Naive & $\overline{N S U}$ & Permissive-Upgrade & Faceted Evaluation \\
\hline $\begin{array}{l}y=\text { true; } \\
z=\text { true; } \\
\text { if }(x) \\
\quad y=\text { false; } \\
\text { if }(y) \\
\quad z=\text { false; } \\
\text { return } z ;\end{array}$ & $\begin{aligned} \mathrm{y}= & \text { true } \\
\mathbf{z}= & \text { true } \\
& - \\
& - \\
p c & =\{\} \\
\mathbf{z}= & \text { false } \\
& -\end{aligned}$ & $\begin{array}{c}\mathrm{y}=\text { true } \\
\mathrm{z}=\text { true } \\
p c=\{k\} \\
\mathrm{y}=\langle k ? \text { false }: \perp\rangle \\
- \\
- \\
-\end{array}$ & $\begin{array}{c}\mathrm{y}=\text { true } \\
\mathrm{z}=\text { true } \\
p c=\{k\} \\
\text { stuck }\end{array}$ & $\begin{array}{c}\mathrm{y}=\text { true } \\
\mathrm{z}=\text { true } \\
p c=\{k\} \\
\mathrm{y}=\langle k ? \text { false }: *\rangle \\
\text { stuck }\end{array}$ & $\begin{array}{c}\mathrm{y}=\text { true } \\
\mathrm{z}=\text { true } \\
p c=\{k\} \\
\mathrm{y}=\langle k ? \text { false }: \text { true }\rangle \\
p c=\{\bar{k}\} \\
\mathrm{z}=\langle k ? \text { true }: \text { false }\rangle \\
-\end{array}$ \\
\hline Return Value: & false & true & & & $\langle k$ ? true : false $\rangle$ \\
\hline
\end{tabular}

branch on $\mathrm{x}$ when $\mathrm{x}=\langle k$ ? false $: \perp\rangle$ is split into separate branches on false and $\perp$. The first test if (false) ... is clearly a no-op, and so is the second test if $(\perp) \ldots$ since if is strict in $\perp$. Since y remains true, the branch on y is taken and so $z$ is set to false. Thus, the function call $\mathrm{f}(\langle k$ ? false $: \perp\rangle)$ returns false.

We now consider the evaluation of $\mathrm{f}(\langle k$ ? true $: \perp\rangle)$ under different dynamic information flow semantics. While the prior semantics that we discuss here have no notion of facets, explaining them in terms of faceted values is illuminating.

Naive An intuitive strategy for handling the assignment $y=f a l s e$ that is conditional on the private input $\mathrm{x}$ is to simply set $\mathrm{y}$ to $\langle k$ ? false $: \perp\rangle$ to reflect that this value depends on private inputs. Unfortunately, this approach is not sound, since it loses the critical information that a public observer should still see y $=$ true. The next conditional branch on y exploits this confusion. Since $\mathrm{y}$ is $\langle k$ ? false : $\perp\rangle$, the branch is not executed, so $\mathrm{z}$ remains true, and so $f(\langle k$ ? true : $\perp\rangle)$ returns true, as illustrated in column 3 . Thus, this naive strategy fails to ensure TINI, since the public output of $f$ leaks the contents of its private input.

Various prior approaches attempt to close this information leak without introducing full faceted values, with mixed results.

No-Sensitive-Upgrade With the no-sensitive-upgrade check [39, 5], execution halts on any attempt to update public variables in code conditional on private data. Under this strategy, the assignment to the public variable $\mathrm{y}$ from code conditional on a private variable $\mathrm{x}$ would get stuck, as shown in the NSU column of Figure 1. This strategy guarantees TINI, but only at the expense of getting stuck on some implicit flows.

Permissive-Upgrade A more flexible approach is to permit the implicit flow caused by the conditional assignment to $\mathrm{y}$, but to record that the analysis has lost track of the correct (original) public facet for $\mathrm{y}$. The Permissive-Upgrade represents this lost information by setting y to the faceted value $\langle k$ ? false : $*\rangle$, where "*" denotes that the public facet is an unknown, non- $\perp$ value. ${ }^{2}$

This permissive upgrade strategy accepts strictly more program executions than the no-sensitive-upgrade approach, but it still resorts to stuck executions in some cases; if the execution ever depends on that missing public facet, then the permissive upgrade strategy halts execution in order to avoid information leaks. In particular, when y is used in the second conditional of Figure 1, the execution gets stuck.

\footnotetext{
${ }^{2}$ The original paper [6] used the false ${ }^{P}$ to represent $\langle k$ ? false : $*\rangle$, where the superscript $P$ denotes "partially leaked".
}

Faceted Evaluation As shown in the last column of Figure 1, faceted values cleanly handle problematic implicit flows. At the conditional assignment to $\mathrm{y}$, the faceted value $\langle k$ ? false : true $\rangle$ simultaneously represents the dual nature of $\mathrm{y}$, which appears false to private observers but true to public observers. Thus, the conditional branch if (y) ... is taken only for public observers, and we record this information by setting the program counter label $p c$ to $\{\bar{k}\}$. Consequently, the assignment $\mathbf{z}=\mathbf{f a l s e}$ updates $\mathbf{z}$ from true to $\langle k$ ? true : false $\rangle$. Critically, this assignment updates only the public facet of $\mathrm{z}$, not its private facet, which stays as true. The final result of the function call is then $\langle k$ ? true : false $\rangle$.

Comparing the behavior of $\mathrm{f}$ on the arguments $\langle k$ ? false : $\perp\rangle$ and $\langle k$ ? true : $\perp\rangle$, we see that, from the perspective of a public observer, $f$ always returns false, correctly reflecting that $f(\perp)$ returns false, and so there is no information leak on this example, despite its problematic implicit flows. Conversely, from the perspective of a private observer authorized to view $f$ 's actual output, $f$ exhibits the correct behavior of returning its private boolean argument.

\section{A Programming Language with Facets}

We formalize faceted evaluation for dynamic information flow in terms of the idealized language $\lambda^{\text {facet }}$ shown in Figure 2. This language extends the $\lambda$-calculus with mutable reference cells, reactive $\mathrm{I} / \mathrm{O}$, a special value $\perp$, and a mechanism for creating faceted values. Despite its intentional minimality, this language captures the essential complexities of dynamic information flow in more realistic languages, since it includes key challenges such as heap allocation, mutation, implicit flows, and higher-order function calls. In particular, conditional tests can be Church-encoded in the usual fashion.

Expressions in $\lambda^{\text {facet }}$ include the standard features of the $\lambda$ calculus, namely variables $(x)$, constants $(c)$, functions $(\lambda x . e)$, and function application $\left(e_{1} e_{2}\right)$. The language also supports mutable reference cells, with operations to create (ref $e$ ), dereference $(! e)$, and update $\left(e_{1}:=e_{2}\right)$ a reference cell. To model JavaScript's interactive nature, $\lambda^{\text {facet }}$ also supports reading from $(\operatorname{read}(f))$ and writing to (write $(f, e)$ ) external resources such as files.

The expression $\left\langle k ? e_{1}: e_{2}\right\rangle$ creates a faceted values where the value of $e_{1}$ is considered secret to principal $k$; observers that cannot see $k$ 's private data will instead see the public facet produced by $e_{2}$. We initially use the terms label and principals as synonyms and focus primarily on confidentiality-Section 5 later introduces integrity labels in the context of robust declassification.

The $\perp$ value is used to represent "nothing", mirroring Smalltalk's nil and JavaScript's undefined. It is primarily used as the public facet in a faceted value $\langle k ? V: \perp\rangle$, which denotes a value $V$ that is private to principal $k$, with no corresponding public value. 
Figure 2: The source language $\lambda^{\text {facet }}$

Syntax:

$\begin{aligned} e::= & \\ & x \\ & c \\ & \lambda x . e \\ & e_{1} e_{2} \\ & \text { ref } e \\ & ! e \\ & e:=e \\ & \operatorname{read}(f) \\ & \text { write }(f, e) \\ & \left\langle k ? e_{1}: e_{2}\right\rangle \\ & \perp\end{aligned}$

$x, y, z$

$c$

$k, l$

$f$

\section{Standard encodings:}

$$
\begin{aligned}
\text { true } & \stackrel{\text { def }}{=} \lambda x \cdot \lambda y \cdot x \\
\text { false } & \stackrel{\text { def }}{=} \lambda x \cdot \lambda y \cdot y \\
\text { if } e_{1} \text { then } e_{2} \text { else } e_{3} & \stackrel{\text { def }}{=}\left(e_{1}\left(\lambda d \cdot e_{2}\right)\left(\lambda d \cdot e_{3}\right)\right)(\lambda x \cdot x) \\
\text { if } e_{1} \text { then } e_{2} & \stackrel{\text { def }}{=} \text { if } e_{1} \text { then } e_{2} \text { else } 0 \\
\text { let } x=e_{1} \text { in } e_{2} & \stackrel{\text { def }}{=}\left(\lambda x \cdot e_{2}\right) e_{1} \\
e_{1} ; e_{2} & \stackrel{\text { def }}{=} \text { let } x=e_{1} \text { in } e_{2}, x \notin F V\left(e_{2}\right)
\end{aligned}
$$

\subsection{Standard Semantics of $\lambda^{\text {facet }}$}

As a point of comparison for our later development, we first present a standard semantics for $\lambda^{\text {facet }}$ that does not handle faceted expressions. In this semantics, values include constants, addresses, closures, and $\perp$, as shown in Figure 3. A closure $(\lambda x . e, \theta)$ is a pair of a $\lambda$-expression and a substitution $\theta$ that maps variables to values. Each reference cell is allocated at an address $a$, and the store $\sigma$ maps addresses to values. The store also maps each file $f$ to a sequence of values $w$. We use the syntax $v . w$ and $w . v$ to indicate a list of values with $v$ as the first or last value, respectively, and use $\emptyset$ to denote both the empty store and the empty substitution.

We formalize the standard semantics via a big-step relation

$$
\sigma, \theta, e \downarrow \sigma^{\prime}, v
$$

that evaluates an expression $e$ in the context of a store $\sigma$ and a substitution $\theta$, and which returns the resulting value $v$ and the (possibly modified) store $\sigma^{\prime}$. This relation is defined via the evaluation rules shown in Figure 3, which are mostly straightforward. For example, the rule [S-APP] evaluates the body of the called function, where the notation $\theta[x:=v]$ denotes the substitution that is identical to $\theta$ except that it maps $x$ to $v$.

The only unusual aspect of this semantics concern the value $\perp$, which essentially means "nothing" or "no information". Operations such as function application, dereference, and assignment are strict in $\perp$; if given a $\perp$ argument they simply return $\perp$ via the various [s-*-BOT] rules. This semantics for $\perp$ facilitates our later use of $\perp$ in faceted values, since, for example, dereferencing a faceted address $\langle k ? a: \perp\rangle$ operates pointwise on the two facets to return a faceted result $\langle k ? v: \perp\rangle$ where $v=\sigma(a)$.

\section{Figure 3: Standard Semantics}

\section{Runtime Syntax}

$\begin{array}{rllll}a & \in & \begin{array}{l}\text { Address } \\ \text { store }\end{array} & = & \begin{array}{l}\left(\text { Address } \rightarrow_{p} \text { value }\right. \\ \left.\text { File } \rightarrow_{\text {value }}\right)\end{array} \\ \theta & \in & \text { subst } & = & \text { Var } \rightarrow_{p} \text { value } \\ v & \in & \text { value } & ::= & c|a|(\lambda x . e, \theta) \mid \perp \\ w & \in & \text { value } & & \end{array}$

Evaluation Rules: $\sigma, \theta, e \downarrow \sigma^{\prime}, v$

$$
\begin{aligned}
& \overline{\sigma, \theta, c \downarrow \sigma, c} \\
& \text { [S-CONST] } \\
& \overline{\sigma, \theta, x \downarrow \sigma, \theta(x)} \\
& \text { [S-VAR] } \\
& \overline{\sigma, \theta,(\lambda x . e) \downarrow \sigma,(\lambda x . e, \theta)} \\
& \sigma, \theta, e_{1} \downarrow \sigma_{1},\left(\lambda x . e, \theta^{\prime}\right) \\
& \sigma_{1}, \theta, e_{2} \downarrow \sigma_{2}, v^{\prime} \\
& \frac{\sigma, \theta^{\prime}\left[x:=v^{\prime}\right], e \downarrow \sigma^{\prime}, v}{\sigma, \theta,\left(e_{1} e_{2}\right) \downarrow \sigma^{\prime}, v} \\
& \sigma, \theta, e_{1} \downarrow \sigma_{1}, \perp \\
& \frac{\sigma_{1}, \theta, e_{2} \downarrow \sigma^{\prime}, v}{\sigma, \theta,\left(e_{1} e_{2}\right) \downarrow \sigma^{\prime}, \perp} \\
& \begin{array}{r}
\sigma, \theta, e \downarrow \sigma^{\prime}, v \\
a \notin \operatorname{dom}\left(\sigma^{\prime}\right)
\end{array} \\
& \overline{\sigma, \theta,(\text { ref } e) \downarrow \sigma^{\prime}[a:=v], a} \\
& \frac{\sigma, \theta, e \downarrow \sigma^{\prime}, a}{\sigma, \theta, ! e \downarrow \sigma^{\prime}, \sigma^{\prime}(a)} \\
& \frac{\sigma, \theta, e \downarrow \sigma^{\prime}, \perp}{\sigma, \theta, ! e \downarrow \sigma^{\prime}, \perp} \\
& \begin{array}{c}
\sigma, \theta, e_{1} \downarrow \sigma_{1}, a \\
\sigma_{1}, \theta, e_{2} \downarrow \sigma_{2}, v
\end{array} \\
& \overline{\sigma, \theta, e_{1}:=e_{2} \downarrow \sigma_{2}[a:=v], v} \\
& \sigma, \theta, e_{1} \downarrow \sigma_{1}, \perp \\
& \frac{\sigma_{1}, \theta, e_{2} \downarrow \sigma_{2}, v}{\sigma, \theta, e_{1}:=e_{2} \downarrow \sigma_{2}, v} \\
& \frac{\sigma(f)=v \cdot w}{\sigma, \theta, \operatorname{read}(f) \downarrow \sigma[f:=w], v} \\
& \frac{\sigma, \theta, e \downarrow \sigma^{\prime}, v}{\sigma, \theta, \operatorname{write}(f, e) \downarrow \sigma^{\prime}\left[f:=\sigma^{\prime}(f) \cdot v\right], v} \\
& \overline{\sigma, \theta, \perp \downarrow \sigma, \perp}
\end{aligned}
$$




\section{Faceted Evaluation}

Having defined the standard semantics of the language, we now extend that semantics with faceted values that dynamically track information flow and which provide noninterference guarantees.

Figure 4 shows the additional runtime syntax needed to support faceted values. We use Initial Capitals to distinguish the new metavariable and domains of the faceted semantics $(V \in$ Value, $\Sigma \in$ Store, $\Theta \in$ Subst) from those of the standard semantics $(v \in$ value, $\sigma \in$ store, $\theta \in$ subst $)$.

Values $V$ now contain faceted values of the form

$$
\left\langle k ? V_{\mathrm{H}}: V_{\mathrm{L}}\right\rangle
$$

which contain both a private facet $V_{\mathrm{H}}$ and a public facet $V_{\mathrm{L}}$. For instance, the value $\langle k$ ? $42: 0\rangle$ indicates that 42 is confidential to the principal $k$, and unauthorized viewers instead see the value 0 . Often, the public facet is set to $\perp$ to denote that there is no intended publicly visible facet. Implicit flows introduce public facets other than $\perp$.

We introduce a program counter label called $p c$ that records when the program counter has been influenced by public or private facets. For example, consider the conditional test

$$
\text { if }(\langle k \text { ? true : false }\rangle) \text { then } e_{1} \text { else } e_{2}
$$

for which our semantics needs to evaluate both $e_{1}$ and $e_{2}$. During the evaluation of $e_{1}$, we add $k$ to $p c$ to record that this computation depends on data private to $k$. Conversely, during the evaluation of $e_{2}$, we add $\bar{k}$ to $p c$ to record that this computation is dependent on the corresponding public facet. Formalizing this idea, we say that a branch $h$ is either a principal $k$ or its negation $\bar{k}$, and that $p c$ is a set of branches. Note that $p c$ can never include both $k$ and $\bar{k}$, since that would reflect a computation dependent on both private and public facets.

The following operation $\left\langle\left\langle p c ? V_{1}: V_{2}\right\rangle\right\rangle$ creates new faceted values, where the resulting value appears like $V_{1}$ to observers that can see the computation corresponding to $p c$, and appears like $V_{2}$ to all other observers.

$$
\begin{array}{lll}
\left\langle\left\langle\emptyset ? V_{n}: V_{o}\right\rangle\right\rangle & \stackrel{\text { def }}{=} & V_{n} \\
\left\langle\left\langle\{k\} \cup \text { rest } ? V_{n}: V_{o}\right\rangle\right\rangle & \stackrel{\text { def }}{=} & \left\langle k ?\left\langle\left\langle\text { rest } ? V_{n}: V_{o}\right\rangle\right\rangle: V_{o}\right\rangle \\
\left\langle\left\langle\{\bar{k}\} \cup \text { rest } ? V_{n}: V_{o}\right\rangle\right\rangle & \stackrel{\text { def }}{=} & \left\langle k ? V_{o}:\left\langle\left\langle\text { rest } ? V_{n}: V_{o}\right\rangle\right\rangle\right\rangle
\end{array}
$$

For example, $\left\langle\left\langle\{k\} ? V_{\mathrm{H}}: V_{\mathrm{L}}\right\rangle\right\rangle$ returns $\left\langle k ? V_{\mathrm{H}}: V_{\mathrm{L}}\right\rangle$, and this operation generalizes to more complex program counter labels. We sometimes abbreviate $\left\langle\left\langle\{k\} ? V_{\mathrm{H}}: V_{\mathrm{L}}\right\rangle\right\rangle$ as $\left\langle\left\langle k ? V_{\mathrm{H}}: V_{\mathrm{L}}\right\rangle\right.$.

We define the faceted value semantics via the big-step evaluation relation:

$$
\Sigma, \Theta, e \Downarrow_{p c} \Sigma^{\prime}, V
$$

that evaluates an expression $e$ in the context of a store $\Sigma$, a substitution $\Theta$, and a program counter label $p c$, and which returns the resulting value $V$ and the (possibly modified) store $\Sigma^{\prime}$.

Rule [F-SPLIT] shows how evaluation of a faceted expression $\left\langle k ? e_{1}: e_{2}\right\rangle$ evaluates both $e_{1}$ and $e_{2}$ to values $V_{1}$ and $V_{2}$, with $p c$ updated appropriately with $k$ and $\bar{k}$ during these two evaluations. The two values are then combined via the operation $\left\langle\left\langle k\right.\right.$ ? $\left.\left.V_{1}: V_{2}\right\rangle\right\rangle$. As an optimization, if the current computation already depends on $k$-private data (i.e., $k \in p c$ ), then rule [F-LEFT] evaluates only $e_{1}$, thus preserving the invariant that $p c$ never contains both $k$ and $\bar{k}$. Conversely, if $\bar{k} \in p c$ then [F-RIGHT] evaluates only $e_{2}$.

Function application $\left(e_{1} e_{2}\right)$ is somewhat tricky, since $e_{1}$ may evaluate to a faceted value tree with closures (or $\perp$ ) at the leaves. To handle this situation, the rule [F-APP] evaluates each $e_{i}$ to a value $V_{i}$ and then delegates to the auxiliary judgement:

$$
\Sigma,\left(V_{1} V_{2}\right) \Downarrow_{p c} \Sigma^{\prime}, V^{\prime}
$$

This auxiliary judgement recursively traverses through any faceted values in $V_{1}$ to perform the actual function applications. If $V_{1}$ is a closure, then rule [FA-FUN] proceeds as normal. If $V_{1}$ is a facet $\left\langle k ? V_{\mathrm{H}}: V_{\mathrm{L}}\right\rangle$, then the rule [FA-SPLIT] applies both $V_{\mathrm{H}}$ and $V_{\mathrm{L}}$ to the argument $V_{2}$, in a manner similar to the rule [F-SPLIT] discussed above. Rules [FA-LEFT] and [FA-RIGHT] are optimized versions of [FA-SPLIT] for cases where $k$ or $\bar{k}$ are already in $p c$. Finally, the "undefined" value $\perp$ can be applied as a function and returns $\perp$ via [FA-BOT] (much like the earlier [S-APP-BOT] rule).

As an example, consider the function application $(f 4)$ where $f$ is a private function represented as $\langle k ?(\lambda x . e): \perp\rangle$. The rules [F-APP] and [FA-SPLIT] decompose the application $(f 4)$ into two separate applications: $((\lambda x . e) 4)$ and $(\perp 4)$. The first application evaluates normally via [FA-FUN] to a result, say $V$, and the second application evaluates to $\perp$ via [FA-BOT], so the result of the call is $\langle k ? V: \perp\rangle$, thus marking the result of the call as private.

The operand of a dereference operation $(! e)$ may also be a faceted value tree. In this case, the rule [F-REF] uses the helper function deref $\left(\Sigma, V_{a}, p c\right)$ to decompose $V_{a}$ into appropriate addresses, retrieve the corresponding values from the store $\Sigma$, and to combine these store values into a new faceted value. As an optimization, any facets in the address $V_{a}$ that are not consistent with $p c$ are ignored.

In a similar manner, the rule [F-ASSIGN] uses the helper function $\operatorname{assign}\left(\Sigma, p c, V_{a}, V\right)$ to decompose $V_{a}$ into appropriate addresses and to update the store $\Sigma$ at those locations with $V$, while ensuring that each update is only visible to appropriate principals that are consistent with $p c$, to avoid information leaks via implicit flows.

The faceted semantics of I/O operations introduces some additional complexities since it involves communication with external, non-faceted files. Each file $f$ has an associated view view $(f)=$ $\left\{k_{1}, \ldots, k_{n}\right\}$ describing which observers may see the contents of that file. The following section defines when a computation with program counter label $p c$ is visible to a view $L$, and also interprets $L$ to project a faceted value $V$ to a non-faceted value $v=L(V)$. We use these two concepts to map between faceted computations and external non-faceted values in files.

A read operation $\operatorname{read}(f)$ may be executed multiple times with different $p c$ labels. Of these multiple executions, only the single execution where $p c$ is visible to view $(f)$ actually reads from the file via [F-READ]; all other executions are no-ops via [F-READ-IGNORE]. The non-faceted value $v$ read from the file is converted to a faceted value $\left\langle\left\langle p c^{\prime} ? v: \perp\right\rangle\right\rangle$ that is only visible to view $(f)$, where $p c^{\prime}$ is the program counter representation of that view.

An output write $(f, e)$ behaves in a similar manner, so only one execution writes to the file via the rule [F-WRITE]. This rule uses the projection operation $v=L(V)$ where $L=\operatorname{view}(f)$ to project the faceted value $V$ produced by $e$ into a corresponding non-faceted value $v$ that is actually written to the file.

For simplicity, we Church-encode conditional branches as function calls, and so the implicit flows caused by conditional branches are a special case of those caused by function calls and are appropriately handled by the various rules in Figure 4. To provide helpful intuition, however, Figure 5 sketches alternative direct rules for evaluating a conditional test if $e_{1}$ then $e_{2}$ else $e_{3}$. In particular, if $e_{1}$ evaluates to a faceted value $\left\langle k ? V_{H}: V_{L}\right\rangle$, the if statement is evaluated potentially twice, using facets $V_{H}$ and $V_{L}$ as the conditional test by the [F-IF-SPLIT] rule.

\subsection{The Projection Property}

Recall that a view is a set of principals $L=\left\{k_{1}, \ldots, k_{n}\right\}$. This view defines what values a particular observer is authorized to see. In particular, an observer with view $L$ sees the private facet $V_{\mathrm{H}}$ in a value $\left\langle k ? V_{\mathrm{H}}: V_{\mathrm{L}}\right\rangle$ only when $k \in L$, and sees $V_{\mathrm{L}}$ otherwise. Thus, each view $L$ serves as a projection function that maps each faceted value $V \in$ Value into a corresponding non-faceted value 


\section{Runtime Syntax}

$\begin{array}{rllll}\Sigma & \in & \text { Store } & = & \left(\text { Address } \rightarrow_{p} \text { Value }\right) \cup\left(\text { File } \rightarrow \text { value }^{*}\right) \\ \Theta & \in & \text { Subst } & = & \text { Var } \rightarrow p \text { Value } \\ R & \in & \text { RawValue } & ::= & c|a|(\lambda x . e, \Theta) \mid \perp \\ V & \in & \text { Value } & ::= & R \mid\left\langle k ? V_{1}: V_{2}\right\rangle \\ h & \in & \text { Branch } & ::= & k \mid \bar{k} \\ p c & \in & P C & = & 2^{\text {Branch }}\end{array}$

Evaluation Rules: $\quad \Sigma, \Theta, e \Downarrow_{p c} \Sigma^{\prime}, V$

$$
\begin{gathered}
\overline{\Sigma, \Theta, c \Downarrow_{p c} \Sigma, c} \\
\overline{\Sigma, \Theta, x \Downarrow_{p c} \Sigma, \Theta(x)} \\
\overline{\Sigma, \Theta,(\lambda x . e) \Downarrow_{p c} \Sigma,(\lambda x . e, \Theta)} \\
\Sigma, \Theta, e_{1} \Downarrow_{p c} \Sigma_{1}, V_{1} \\
\Sigma_{1}, \Theta, e_{2} \Downarrow_{p c} \Sigma_{2}, V_{2} \\
\frac{\Sigma_{2},\left(V_{1} V_{2}\right) \Downarrow_{p c} \Sigma^{\prime}, V^{\prime}}{\Sigma, \Theta,\left(e_{1} e_{2}\right) \Downarrow_{p c} \Sigma^{\prime}, V^{\prime}} \\
\notin p c \quad \Sigma, \Theta, e_{1} \Downarrow_{p c \cup\{k\}} \Sigma_{1}, \\
\notin p c \quad \Sigma_{1}, \Theta, e_{2} \Downarrow_{p c \cup\{\bar{k}\}} \Sigma_{2}, V_{2} \\
\frac{k \in p c \quad \Sigma, \Theta, e_{1} \Downarrow_{p c} \Sigma^{\prime}, V}{\Sigma, \Theta,\left\langle k ? e_{1}: e_{2}\right\rangle \Downarrow_{p c} \Sigma^{\prime}, V} \\
\frac{\bar{k} \in p c \quad \Sigma, \Theta, e_{2} \Downarrow_{p c} \Sigma^{\prime}, V}{\Sigma, \Theta,\left\langle k ? e_{1}: e_{2}\right\rangle \Downarrow_{p c} \Sigma^{\prime}, V} \\
\frac{\left\langle k ? e_{1}: e_{2}\right\rangle \Downarrow_{p c} \Sigma_{2},\left\langle\left\langle k ? V_{1}:\right.\right.}{\Sigma, \Theta, \perp \Downarrow_{p c} \Sigma, \perp}
\end{gathered}
$$

[F-CONST]$$
\text { [F-VAR] }
$$

[F-APP]

\begin{tabular}{ccc}
$k \notin p c$ & $\Sigma, \Theta, e_{1} \Downarrow_{p c \cup\{k\}} \Sigma_{1}, V_{1}$ \\
$\bar{k} \notin p c$ & $\Sigma_{1}, \Theta, e_{2} \Downarrow_{p c \cup\{\bar{k}\}} \Sigma_{2}, V_{2}$ \\
\hline$\Sigma, \Theta,\left\langle k ? e_{1}: e_{2}\right\rangle \Downarrow_{p c} \Sigma_{2},\left\langle\left\langle k ? V_{1}: V_{2}\right\rangle\right\rangle$
\end{tabular}

[F-SPLIT]

[F-FUN]

$$
\frac{\Sigma, \Theta, e \Downarrow_{p c} \Sigma^{\prime}, V \quad V^{\prime}=\operatorname{deref}\left(\Sigma^{\prime}, V, p c\right)}{\Sigma, \Theta, ! e \Downarrow_{p c} \Sigma^{\prime}, V^{\prime}}
$$

[F-REF]

$$
\begin{gathered}
a \notin \operatorname{dom}\left(\Sigma^{\prime}\right) \\
V=\left\langle\left\langle p c ? V^{\prime}: \perp\right\rangle\right\rangle \\
\Sigma, \Theta,(\operatorname{ref} e) \Downarrow p c \Sigma^{\prime}[a:=V], a
\end{gathered}
$$

[F-DEREF]

$$
\begin{gathered}
\Sigma, \Theta, e_{1} \Downarrow_{p c} \Sigma_{1}, V_{1} \\
\Sigma_{1}, \Theta, e_{2} \Downarrow_{p c} \Sigma_{2}, V^{\prime} \\
\frac{\Sigma^{\prime}=\operatorname{assign}\left(\Sigma_{2}, p c, V_{1}, V^{\prime}\right)}{\Sigma, \Theta, e_{1}:=e_{2} \Downarrow_{p c}, V^{\prime}}
\end{gathered}
$$

[F-ASSIGN]

$$
\Sigma(f)=v . w \quad L=\text { view }(f)
$$$$
\frac{p c \text { visible to } L \quad p c^{\prime}=L \cup\{\bar{k} \mid k \notin L\}}{\Sigma, \Theta, \operatorname{read}(f) \Downarrow_{p c} \Sigma[f:=w],\left\langle\left\langle p c^{\prime} ? v: \perp\right\rangle\right\rangle}
$$

[F-READ]

$$
\frac{p c \text { not visible to view }(f)}{\Sigma, \Theta, r e a d(f) \text { t }}
$$

[F-LEFT]

$$
\overline{\Sigma, \Theta, \operatorname{read}(f) \Downarrow_{p c} \Sigma, \perp}
$$

[F-READ-IGNORE]

$$
\begin{array}{rl}
\Sigma, \Theta, e \Downarrow_{p c} \Sigma^{\prime}, V & p c \text { visible to view }(f) \\
L=\text { view }(f) & v=L(V)
\end{array}
$$

[F-RIGHT]

$\overline{\Sigma, \Theta, \text { write }(f, e) \Downarrow p c \Sigma^{\prime}\left[f:=\Sigma^{\prime}(f) \cdot v\right], V}$

[F-WRITE]

$\frac{\Sigma, \Theta, e \Downarrow_{p c} \Sigma^{\prime}, V \quad p c \text { not visible to view }(f)}{\Sigma, \Theta, w r i t t e(f, e) \Downarrow_{p c} \Sigma^{\prime}, V}$

[F-WRITE-IGNORE]

Application Rules $\Sigma,\left(V_{1} V_{2}\right) \Downarrow_{p c} \Sigma^{\prime}, V^{\prime}$

$$
\begin{aligned}
& \Sigma, \Theta[x:=V], e \Downarrow_{p c} \Sigma^{\prime}, V^{\prime} \\
& \overline{\Sigma,((\lambda x . e, \Theta) V) \Downarrow_{p c} \Sigma^{\prime}, V^{\prime}} \\
& k \notin p c \quad \Sigma,\left(V_{\mathrm{H}} V_{2}\right) \Downarrow_{p c \cup\{k\}} \Sigma_{1}, V_{\mathrm{H}}^{\prime} \\
& \bar{k} \notin p c \quad \Sigma_{1},\left(V_{\mathrm{L}} V_{2}\right) \Downarrow_{p c \cup\{\bar{k}\}} \Sigma^{\prime}, V_{\mathrm{L}}^{\prime} \\
& \Sigma \overline{\left(\left\langle k ? V_{\mathrm{H}}: V_{\mathrm{L}}\right\rangle V_{2}\right) \Downarrow_{p c} \Sigma^{\prime},\left\langle\left\langle k ? V_{\mathrm{H}}^{\prime}: V_{\mathrm{L}}^{\prime}\right\rangle\right\rangle}
\end{aligned}
$$

[FA-FUN]

$$
\begin{aligned}
& \frac{k \in p c \quad \Sigma,\left(V_{\mathrm{H}} V_{2}\right) \Downarrow_{p c} \Sigma^{\prime}, V}{\Sigma,\left(\left\langle k ? V_{\mathrm{H}}: V_{\mathrm{L}}\right\rangle V_{2}\right) \Downarrow_{p c} \Sigma^{\prime}, V} \\
& \frac{\bar{k} \in p c \quad \Sigma,\left(V_{\mathrm{L}} V_{2}\right) \Downarrow_{p c} \Sigma^{\prime}, V}{\Sigma,\left(\left\langle k ? V_{\mathrm{H}}: V_{\mathrm{L}}\right\rangle V_{2}\right) \Downarrow_{p c} \Sigma^{\prime}, V}
\end{aligned}
$$

[FA-LEFT]

[FA-RIGHT]

$$
\overline{\Sigma,(\perp V) \Downarrow_{p c} \Sigma, \perp}
$$

$$
\begin{aligned}
& \text { deref : Store } \times \text { Value } \times P C \\
& \text { deref }(\Sigma, a, p c) \\
& \text { deref }(\Sigma, \perp, p c) \\
& \operatorname{deref}\left(\Sigma,\left\langle k ? V_{\mathrm{H}}: V_{\mathrm{L}}\right\rangle, p c\right) \\
& \text { assign : Store } \times P C \times \text { Value } \times \text { Value } \\
& \operatorname{assign}(\Sigma, p c, a, V) \\
& \operatorname{assign}(\Sigma, p c, \perp, V) \\
& \operatorname{assign}\left(\Sigma, p c,\left\langle k ? V_{\mathrm{H}}: V_{\mathrm{L}}\right\rangle, V\right)
\end{aligned}
$$

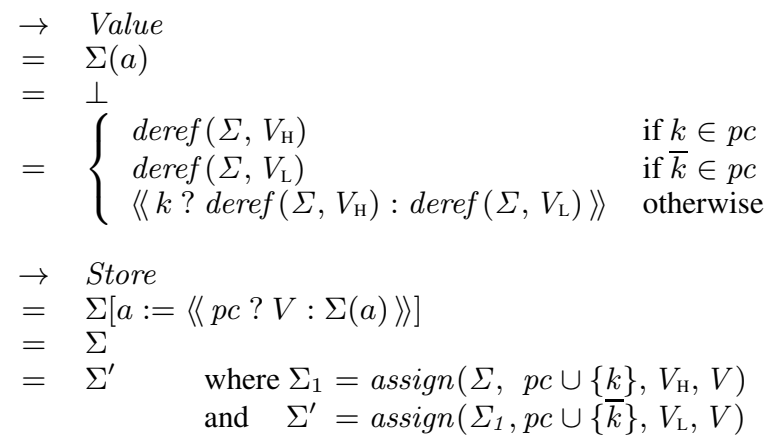


Figure 5: Faceted Evaluation Semantics for Derived Encodings

$$
\begin{aligned}
& \Sigma, \Theta, e_{1} \Downarrow_{p c} \Sigma_{1} \text {, true } \quad \Sigma_{1}, \Theta, e_{2} \Downarrow_{p c} \Sigma^{\prime}, V \\
& \Sigma, \Theta \text {, if } e_{1} \text { then } e_{2} \text { else } e_{3} \Downarrow_{p c} \Sigma^{\prime}, V \quad \text { [F-IF-TRUE] } \\
& \frac{\Sigma, \Theta, e_{1} \Downarrow_{p c} \Sigma_{1}, \text { false } \quad \Sigma_{1}, \Theta, e_{3} \Downarrow_{p c} \Sigma^{\prime}, V}{\Sigma, \Theta, \text { if } e_{1} \text { then } e_{2} \text { else } e_{3} \Downarrow_{p c} \Sigma^{\prime}, V} \quad \text { [F-IF-FALSE] } \\
& \frac{\Sigma, \Theta, e_{1} \Downarrow_{p c} \Sigma^{\prime}, \perp}{\Sigma, \Theta, \text { if } \perp \text { then } e_{2} \text { else } e_{3} \Downarrow_{p c} \Sigma^{\prime}, \perp} \quad \text { [F-IF-BOT] } \\
& \Sigma, \Theta, e_{1} \Downarrow_{p c} \Sigma_{1},\left\langle k ? V_{H}: V_{L}\right\rangle \\
& e_{H}=\text { if } V_{H} \text { then } e_{2} \text { else } e_{3} \\
& e_{L}=\text { if } V_{L} \text { then } e_{2} \text { else } e_{3} \\
& \Sigma_{1}, \Theta,\left\langle k ? e_{H}: e_{L}\right\rangle \Downarrow_{p c} \Sigma^{\prime}, V \\
& \overline{\Sigma, \Theta}, \text { if } e_{1} \text { then } e_{2} \text { else } e_{3} \Downarrow_{p c} \Sigma^{\prime}, V \text { [F-IF-SPLIT] }
\end{aligned}
$$

of the standard semantics:

$$
\begin{aligned}
L: \text { Value } & \rightarrow \text { value } \\
L\left(\left\langle k ? V_{1}: V_{2}\right\rangle\right) & = \begin{cases}L\left(V_{1}\right) & \text { if } k \in L \\
L\left(V_{2}\right) & \text { if } k \notin L\end{cases} \\
L(c) & =c \\
L(a) & =a \\
L(\perp) & =\perp \\
L((\lambda x . e, \Theta)) & =(\lambda x . L(e), L(\Theta))
\end{aligned}
$$

We extend $L$ to also project faceted substitutions $\Theta \in$ Subst and stores $\Sigma \in$ Store into non-faceted substitutions and stores of the standard semantics. A file $f$ is visible only to view $(f)$, and appears empty $(\epsilon)$ to all other views.

$$
\begin{aligned}
& L: \text { Subst } \rightarrow \text { subst } \\
& L(\Theta)=\lambda x \cdot L(\Theta(x)) \\
& L: \text { Store } \rightarrow \text { store } \\
& L(\Sigma)=\lambda a \cdot L(\Sigma(a)) \\
& \cup \quad \lambda f . \begin{cases}\Sigma(f) & \text { if } L=\text { view }(f) \\
\epsilon & \text { otherwise }\end{cases}
\end{aligned}
$$

We also use a view $L$ to operate on expressions, where this operation eliminates faceted expressions and also performs access control on I/O operations by eliminating accesses to files that are not authorized under that view:

$$
\begin{aligned}
L: \text { Expr (with facets) } & \rightarrow \text { Expr (without facets) } \\
L\left(\left\langle k ? e_{1}: e_{2}\right\rangle\right) & =\left\{\begin{array}{l}
L\left(e_{1}\right) \text { if } k \in L \\
L\left(e_{2}\right) \text { if } k \notin L \\
\operatorname{read}(f) \text { if } L=\operatorname{view}(f) \\
\perp \quad \text { otherwise } \\
\text { write }(f, L(e)) \text { if } L=\text { view }(f) \\
L(e) \text { otherwise }
\end{array}\right. \\
L(\text { write }(f, e)) & =\text { compatible closure }
\end{aligned}
$$

Thus, views naturally serve as a projection from each domain of the faceted semantics into a corresponding domain of the standard semantics. We now use these views-as-projections to formalize the relationship between these two semantics.

A computation with program counter label $p c$ is considered visible to a view $L$ only when the principals mentioned in $p c$ are consistent with $L$, in the sense that:

$$
\begin{aligned}
& \forall k \in p c, k \in L \\
& \forall \bar{k} \in p c, k \notin L
\end{aligned}
$$

We first show that the operation $\left\langle\left\langle p c ? V_{1}: V_{2}\right\rangle\right\rangle$ has the expected behavior, in that from the perspective of a view $L$, it appears to return $V_{1}$ only when $p c$ is visible to $L$, and appears to return $V_{2}$ otherwise.

Lemma 1. If $V=\left\langle\left\langle p c\right.\right.$ ? $\left.\left.V_{1}: V_{2}\right\rangle\right\rangle$ then

$$
L(V)= \begin{cases}V_{1} & \text { if pc is visible to } L \\ V_{2} & \text { otherwise }\end{cases}
$$

We next show that the auxiliary functions deref and assign exhibit the expected behavior when projected under a view $L$. First, if deref $(\Sigma, V)$ returns $V^{\prime}$, then the projected result $L\left(V^{\prime}\right)$ is a non-faceted value that is identical to first projecting the store $L(\Sigma)$, projecting the target address $L(V)$, and then dereferencing the projected store at the projected address $L(\Sigma)(L(V))$.

Lemma 2. If $V^{\prime}=\operatorname{deref}(\Sigma, V, p c)$ then $L\left(V^{\prime}\right)=L(\Sigma)(L(V))$.

Next, from the perspective of any view $L$, if $p c$ is visible to $L$ then the operation $\operatorname{assign}\left(\Sigma, p c, V_{1}, V_{2}\right)$ appears to update the address $L\left(V_{1}\right)$ appropriately. Conversely, if $p c$ is not visible to $L$, then this operation has no observable effect.

Lemma 3. If $\Sigma^{\prime}=\operatorname{assign}\left(\Sigma, p c, V_{1}, V_{2}\right)$ then

$$
L\left(\Sigma^{\prime}\right)= \begin{cases}L(\Sigma)\left[L\left(V_{1}\right):=L\left(V_{2}\right)\right] & \text { if pc is visible to } L \\ L(\Sigma) & \text { otherwise }\end{cases}
$$

A consequence of Lemma 3 is that evaluation with a $p c$ that is not visible to a view $L$ produces no observable change in the store.

Lemma 4. Suppose pc is not visible to $L$ and that

$$
\Sigma, \Theta, e \Downarrow_{p c} \Sigma^{\prime}, V
$$

Then $L(\Sigma)=L\left(\Sigma^{\prime}\right)$

Proof. In the auxiliary material for this paper.

We now prove our central projection theorem showing that an evaluation under the faceted semantics is equivalent to many evaluations under the standard semantics, one for each possible view for which $p c$ is visible.

Theorem 1 (Projection Theorem). Suppose

$$
\Sigma, \Theta, e \Downarrow_{p c} \Sigma^{\prime}, V
$$

Then for any view $L$ for which $p c$ is visible,

$$
L(\Sigma), L(\Theta), L(e) \downarrow L\left(\Sigma^{\prime}\right), L(V)
$$

Proof. In the auxiliary material for this paper.

Consequently, if $p c$ is initially empty, then faceted evaluation simulates $2^{n}$ standard evaluations, where $n$ is the number of principals.

\subsection{Termination-Insensitive Non-Interference}

The projection property enables a very simple proof of noninterference; it already captures the idea that information from one view does not leak into an incompatible view, since the projected computations are independent. To formalize this argument, we start by defining two faceted values to be $L$-equivalent if they have identical standard values for view $L$. This notion of $L$-equivalence naturally extends to substitutions $\left(\Theta_{1} \sim_{L} \Theta_{2}\right)$ and stores $\left(\Sigma_{1} \sim_{L} \Sigma_{2}\right)$ :

$$
\begin{array}{ccc}
\left(V_{1} \sim_{L} V_{2}\right) & \text { iff } & L\left(V_{1}\right)=L\left(V_{2}\right) \\
\left(\Theta_{1} \sim_{L} \Theta_{2}\right) & \text { iff } & L\left(\Theta_{1}\right)=L\left(\Theta_{2}\right) \\
\left(\Sigma_{1} \sim_{L} \Sigma_{2}\right) & \text { iff } & L\left(\Sigma_{1}\right)=L\left(\Sigma_{2}\right)
\end{array}
$$

Together with the Projection Theorem, this notion of $L$-equivalence enables us to conveniently state and prove the standard correctness property of termination-insensitive non-interference. 
Figure 6: Efficient Construction of Faceted Values

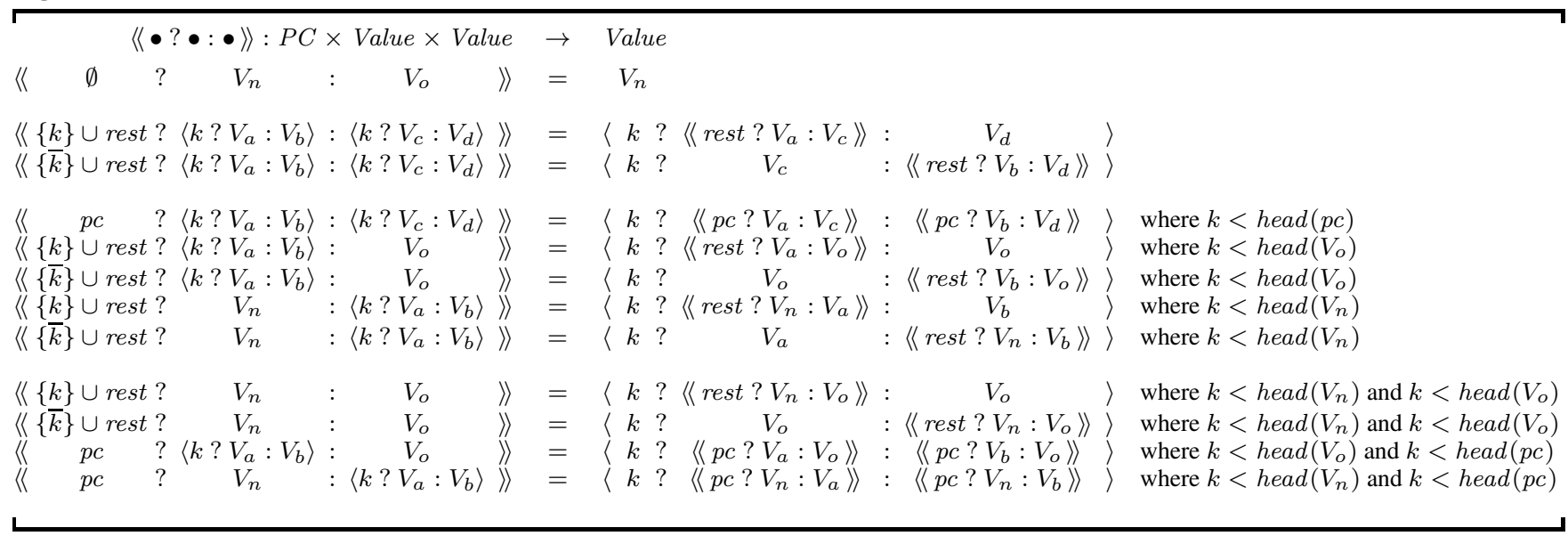

Theorem 2 (Termination-Insensitive Non-Interference). Let $L$ be any view. Suppose

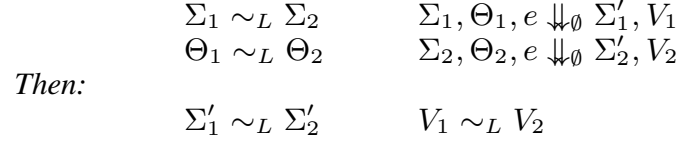

Proof. By the Projection Theorem:

$$
\begin{aligned}
& L\left(\Sigma_{1}\right), L\left(\Theta_{1}\right), L(e) \downarrow L\left(\Sigma_{1}^{\prime}\right), L\left(V_{1}\right) \\
& L\left(\Sigma_{2}\right), L\left(\Theta_{2}\right), L(e) \downarrow L\left(\Sigma_{2}^{\prime}\right), L\left(V_{2}\right)
\end{aligned}
$$

The $L$-equivalence assumptions imply that $L\left(\Theta_{1}\right)=L\left(\Theta_{2}\right)$ and $L\left(\Sigma_{1}\right)=L\left(\Sigma_{2}\right)$. Hence $L\left(\Sigma_{1}^{\prime}\right)=L\left(\Sigma_{2}^{\prime}\right)$ and $L\left(V_{1}\right)=L\left(V_{2}\right)$ since the standard semantics is deterministic.

This theorem can be generalized to computations with arbitrary program counter labels, but then non-interference holds only for views for which that $p c$ is visible.

\subsection{Efficient Construction of Faceted Values}

The definition of the operation $\left\langle\left\langle p c ? V_{1}: V_{2}\right\rangle\right\rangle$ presented above is optimized for clarity, but may result in a suboptimal representation for faceted values. For instance, the operation $\langle\langle\{k\}$ ? $\langle k ? 1: 0\rangle$ : $2\rangle\rangle$ returns the faceted value tree $\langle k ?\langle k ? 1: 0\rangle: 2\rangle$ containing a dead facet 0 that is not visible in any view. We now present an optimized version of this operation that avoids introducing dead facets.

The essential idea is to introduce a fixed total ordering on principals and to ensure that in any faceted value tree, the path from the root to any leaf only mentions principals in a strictly increasing order. In order to maintain this ordering, we introduce a head function that returns the lowest label in a value or program counter, or a result $\infty$ that is considered higher than any label.

$$
\begin{aligned}
\text { head }: \text { Value } & \rightarrow \text { Label } \cup\{\infty\} \\
\text { head }\left(\left\langle k ? V_{1}: V_{2}\right\rangle\right) & =k \\
\text { head }(R) & =\infty \\
\text { head }: P C & \rightarrow \text { Label } \cup\{\infty\} \\
\operatorname{head}(\{k\} \cup \text { rest }) & =k \quad \text { if } \forall k^{\prime} \text { or } \overline{k^{\prime}} \in \text { rest. } k<k^{\prime} \\
\text { head }(\{\bar{k}\} \cup \text { rest }) & =k \quad \text { if } \forall k^{\prime} \text { or } \overline{k^{\prime}} \in \text { rest. } k<k^{\prime} \\
\text { head }(\{\}) & =\infty
\end{aligned}
$$

Figure 6 redefines the facet-construction operation to build values respecting the ordering of labels. The definition is verbose but straightforward; it performs a case analysis to identify the smallest possible label $k$ to put at the root of the newly created value. The revised definition still satisfies the specification provided by Lemma 1.

\section{Comparison to Prior Semantics}

Prior work presented the no-sensitive-upgrade (NSU) semantics [39, 5] and the permissive-upgrade (PU) semantics [6] for dynamic information flow. In this section, we adapt both of these semantics to our notation to illustrate how faceted evaluation extends both of these prior techniques. For clarity, in this section we assume that there is only a single principal $k$ and omit I/O operations, since the two prior semantics were formalized under these assumptions. Finally, we use the optimized facet-construction operation from Figure 6 in order to avoid reasoning about dead facets.

\subsection{Comparison to No-Sensitive-Upgrade Semantics}

We formalize the NSU semantics via the evaluation relation

$$
\Sigma, \Theta, e \Downarrow_{p c} \Sigma^{\prime}, V
$$

defined by the [NSU-*] rules in Figure 7 . These rules are somewhat analogous to the faceted evaluation rules of Figure 4, but with some noticeable limitations and restrictions. In particular, the NSU semantics marks each raw value $R$ as being either public or private:

$$
\begin{array}{ccc}
V::= & R & \text { public values } \\
\mid & \langle k ? R: \perp\rangle & \text { private values }
\end{array}
$$

The NSU semantics cannot record any public facet other than $\perp$. The faceted value $\langle k ? R: \perp\rangle$ is traditionally written simply as $R^{k}$ in prior semantics, denoting that $R$ is private to principal $k$, with no representation for a corresponding public facet. This restriction on values means that the NSU semantics never needs to split the computation in the manner performed by the earlier [F-SPLIT] and [FA-SPLIT] rules. Instead, applications of a private closure $\left\langle k ?\left(\lambda x . e, \Theta^{\prime}\right): \perp\right\rangle$ extends the program counter $p c$ with the label $k$ during the call, reflecting that this computation is dependent on $k$-private data. Thus, under the NSU semantics, the program counter label is simply a set of principals, and never contains negated principals $\bar{k}$.

$$
p c \in P C=2^{\text {Label }}
$$

After the callee returns a result $V$, the following operation $\langle k\rangle^{p c} V$ creates a faceted value semantically equivalent to $\langle k ? V: \perp\rangle$, with 
Figure 7: No Sensitive Upgrade Semantics

NSU Evaluation Rules: $\Sigma, \Theta, e \Downarrow_{p c} \Sigma^{\prime}, V$

$$
\begin{aligned}
& \overline{\Sigma, \Theta, c \Downarrow_{p c} \Sigma, c} \\
& \overline{\Sigma, \Theta, x \Downarrow_{p c} \Sigma, \Theta(x)} \\
& \overline{\Sigma, \Theta,(\lambda x . e) \Downarrow_{p c} \Sigma,(\lambda x . e, \Theta)} \\
& \overline{\Sigma, \Theta, \perp \Downarrow_{p c} \Sigma, \perp} \\
& \frac{\Sigma, \Theta, e \Downarrow_{p c \cup\{k\}} \Sigma^{\prime}, V}{\Sigma, \Theta,\langle k ? e: \perp\rangle \Downarrow_{p c} \Sigma^{\prime},\langle k\rangle^{p c} V} \\
& \Sigma, \Theta, e_{1} \Downarrow_{p c} \Sigma_{1},\left(\lambda x . e, \Theta^{\prime}\right) \\
& \Sigma_{1}, \Theta, e_{2} \Downarrow_{p c} \Sigma_{2}, V^{\prime} \\
& \frac{\Sigma, \Theta^{\prime}\left[x:=V^{\prime}\right], e \Downarrow_{p c} \Sigma^{\prime}, V}{\Sigma, \Theta,\left(e_{1} e_{2}\right) \Downarrow_{p c} \Sigma^{\prime}, V} \quad \text { [NSU-APP] } \\
& \Sigma, \Theta, e_{1} \Downarrow_{p c} \Sigma_{1}, \perp \\
& \Sigma_{1}, \Theta, e_{2} \Downarrow_{p c} \Sigma_{2}, V^{\prime} \\
& \overline{\Sigma, \Theta,\left(e_{1} e_{2}\right) \Downarrow_{p c} \Sigma^{\prime}, \perp} \\
& \Sigma, \Theta, e_{1} \Downarrow_{p c} \Sigma_{1},\left\langle k ?\left(\lambda x . e, \Theta^{\prime}\right): \perp\right\rangle \\
& \Sigma_{1}, \Theta, e_{2} \Downarrow_{p c} \Sigma_{2}, V^{\prime} \\
& \frac{\Sigma, \Theta^{\prime}\left[x:=V^{\prime}\right], e \Downarrow_{p c \cup\{k\}} \Sigma^{\prime}, V}{\Sigma, \Theta,\left(e_{1} e_{2}\right) \Downarrow_{p c} \Sigma^{\prime},\langle k\rangle^{p c} V} \quad \text { [NSU-APP-K] } \\
& \Sigma, \Theta, e \Downarrow_{p c} \Sigma^{\prime}, V^{\prime} \\
& a \notin \operatorname{dom}\left(\Sigma^{\prime}\right) \\
& V=\left\langle\left\langle p c ? V^{\prime}: \perp\right\rangle\right\rangle \\
& \overline{\Sigma, \Theta,(\text { ref } e) \Downarrow_{p c} \Sigma^{\prime}[a:=V], a} \\
& \Sigma, \Theta, e \Downarrow_{p c} \Sigma^{\prime}, V_{a} \\
& \frac{V=\operatorname{deref}\left(\Sigma^{\prime}, V_{a}, p c\right)}{\Sigma, \Theta, ! e \Downarrow_{p c} \Sigma^{\prime}, V} \\
& \Sigma, \Theta, e_{1} \Downarrow_{p c} \Sigma_{1}, \perp \\
& \frac{\Sigma_{1}, \Theta, e_{2} \Downarrow_{p c} \Sigma_{2}, V}{\Sigma, \Theta, e_{1}:=e_{2} \Downarrow_{p c} \Sigma_{2}, V} \\
& \Sigma, \Theta, e_{1} \Downarrow_{p c} \Sigma_{1}, a \\
& \Sigma_{1}, \Theta, e_{2} \Downarrow_{p c} \Sigma^{\prime}, V \\
& p c=\text { label }\left(\Sigma^{\prime}(a)\right) \\
& \frac{V^{\prime}=\langle\langle p c ? V: \perp\rangle\rangle}{\Sigma, \Theta, e_{1}:=e_{2} \Downarrow_{p c} \Sigma^{\prime}\left[a:=V^{\prime}\right], V} \\
& \Sigma, \theta, e_{1} \Downarrow_{p c} \Sigma_{1},\langle k ? a: \perp\rangle \\
& \Sigma_{1}, \Theta, e_{2} \Downarrow_{p c} \Sigma^{\prime}, V \\
& p c \cup\{k\} \subseteq \operatorname{label}\left(\Sigma^{\prime}(a)\right) \\
& \frac{V^{\prime}=\langle\langle p c ? V: \perp\rangle\rangle}{\Sigma, \Theta, e_{1}:=e_{2} \Downarrow_{p c} \Sigma^{\prime}\left[a:=V^{\prime}\right], V}
\end{aligned}
$$

Figure 8: Permissive Upgrade Semantics (extends Figure 7)

\begin{tabular}{c}
\hline PU Evaluation Rules: $\overline{\Sigma, \Theta, e \Downarrow_{p c} \Sigma^{\prime}, V}$ \\
$\Sigma, \Theta, e_{1} \Downarrow_{p c} \Sigma_{1}, a$ \\
$\Sigma_{1}, \Theta, e_{2} \Downarrow_{p c} \Sigma^{\prime}, V$ \\
$V^{\prime}=\langle\langle p c ? V: *\rangle\rangle$ \\
$\frac{}{\Sigma, \Theta, e_{1}:=e_{2} \Downarrow_{p c} \Sigma^{\prime}\left[a:=V^{\prime}\right], V}$ \\
$\Sigma, \theta, e_{1} \Downarrow_{p c} \Sigma_{1},\langle k ? a: \perp\rangle$ \\
$\Sigma_{1}, \Theta, e_{2} \Downarrow_{p c} \Sigma^{\prime}, V$ \\
$V^{\prime}=\langle\langle p c ? V: *\rangle\rangle$ \\
\hline$\Sigma, \Theta, e_{1}:=e_{2} \Downarrow_{p c} \Sigma^{\prime}\left[a:=V^{\prime}\right], V$ \\
\hline
\end{tabular}

the optimization that the label $k$ is unnecessary if it is subsumed by $p c$ or if it is already in $V$ :

$$
\begin{array}{lll}
\langle k\rangle^{\{k\}} V & = \\
\langle k\rangle^{\{\}} R & =\langle k ? R: \perp\rangle \\
\langle k\rangle^{p c}\langle k ? R: \perp\rangle & =\langle k ? R: \perp\rangle
\end{array}
$$

(This optimization corresponds to the [FA-LEFT] and [FA-RIGHT] rules of the faceted semantics.)

In order to preserve the NSU restriction on values, the NSU semantics needs to carefully restrict assignment statements. Essentially, the NSU evaluation rules for assignment statements halt execution in exactly those situations where the faceted semantics would introduce a non-trivial public facet. These rules use the following function to extract the principals in a value:

$$
\begin{aligned}
& \text { label: Value } \rightarrow P C \\
& \text { label }(\langle k ? R: \perp\rangle)=\{k\} \\
& \operatorname{label}(R)=\emptyset
\end{aligned}
$$

The rule [NSU-ASSIGN] checks that $p c$ is equal to the label on the original value $\Sigma^{\prime}(a)$ of the target location $a$. If this condition holds, then the value $\langle\langle p c ? V: \perp\rangle\rangle$ stored by [NSU-ASSIGN] is actually equal to the value $\left\langle\left\langle p c\right.\right.$ ? $\left.\left.V: \Sigma^{\prime}(a)\right\rangle\right\rangle$ that the faceted semantics would store. Thus, this no-sensitive-upgrade check detects situations where the NSU semantics can avoid information leaks without introducing non- $\perp$ public facets. The rule [NSU-ASSIGN-K] handles assignments where the target address is private $\langle k ? a: \perp\rangle$ in a similar manner to [NSU-ASSIGN].

Because of these no-sensitive-upgrade checks, the NSU semantics will get stuck at precisely the points where the faceted value semantics will create non- $\perp$ public facets. An example of this stuck execution is shown in the $N S U$ column of Figure 1. When the value for $y$ is updated in a context dependent on the confidential value of $\mathrm{x}$, execution gets stuck to prevent loss of information.

If the NSU semantics runs to completion on a given program, then the faceted semantics will produce the same results.

Theorem 3 (Faceted evaluation generalizes NSU evaluation). If $\Sigma, \Theta, e \Downarrow_{p c} \Sigma^{\prime}, V$ then $\Sigma, \Theta, e \Downarrow_{p c} \Sigma^{\prime}, V$.

Proof. In the auxiliary material for this paper.

\subsection{Permissive Upgrades}

The limitations of the NSU semantics motivated the development of a more expressive permissive upgrade (PU) semantics, which reduced (but did not eliminate) stuck executions [6]. Essentially, the PU semantics works by tracking partially leaked data, which 
Figure 9: Declassification of Faceted Values

\section{Declassification Rule}

$$
\begin{gathered}
\Sigma, \Theta, e \Downarrow_{p c} \Sigma^{\prime}, V \\
\mathrm{U}^{P} \notin p c \\
\frac{V^{\prime}=\text { downgrade }_{P}(V)}{\Sigma, \Theta, \text { declassify }_{P} e \Downarrow_{p c} \Sigma^{\prime}, V^{\prime}} \quad \text { [F-DECLASSIFY] }
\end{gathered}
$$

\section{Downgrade Function}

$$
\begin{aligned}
& \text { downgrade }_{P} \text { : Value } \\
& \begin{array}{ll}
\text { downgrade }_{P}(R) & =R \\
\text { downgrade }_{P}\left(\left\langle\mathrm{~S}^{P} ? V_{1}: V_{2}\right\rangle\right) & =\left\langle\mathrm{U}^{P} ?\left\langle\mathrm{~S}^{P} ? V_{1}: V_{2}\right\rangle: V_{1}\right\rangle
\end{array} \\
& \text { downgrade }_{P}\left(\left\langle\mathrm{U}^{P} \text { ? } V_{1}: V_{2}\right\rangle\right)=\left\langle\left\langle\mathrm{U}^{P} \text { ? } V_{1}: \text { downgrade }_{P}\left(V_{2}\right)\right\rangle\right. \\
& \text { downgrade }_{P}\left(\left\langle l ? V_{1}: V_{2}\right\rangle\right) \\
& \left\langle l \text { ? downgrade }{ }_{P}\left(V_{1}\right): \text { downgrade }_{P}\left(V_{2}\right)\right\rangle
\end{aligned}
$$

we represent here as a faceted value $\langle k ? R: *\rangle .^{3}$

$$
\begin{aligned}
& V \quad:=\quad R \quad \text { public values } \\
& \langle k ? R: \perp\rangle \quad \text { private values } \\
& \langle k ? R: *\rangle \quad \text { partially leaked values }
\end{aligned}
$$

Since the public facet is not actually stored, the PU semantics can never use partially leaked values in situations where the public facet is needed, and so partially leaked values cannot be assigned, invoked, or used as a conditional test. In particular, PU computations never need to "split" executions, and so avoid the complexities and expressiveness of faceted evaluation.

We formalize the PU semantics by extending the NSU evaluation relation $\Sigma, \Theta, e \Downarrow_{p c} \Sigma^{\prime}, V$ with the two additional rules shown in Figure 8. The new assignment rules leverage faceted values to handle the complexity involved in tracking partially leaked data. Specifically, if values are stored to a public reference cell in a highsecurity context, the data is partially leaked, and a new faceted value with a non- $\perp$ public facet is created.

Critically, there are no rules for applying partially leaked functions or assigning to partially leaked addresses, and consequently execution gets stuck at these points, corresponding to the explicit checks for partially leaked labels in the original PU semantics [6].

Faceted values subsume the permissive upgrade strategy. The permissive upgrade strategy gets stuck at the points where a faceted value with a non- $\perp$ facet is either applied or used in assignment.

Theorem 4 (Faceted evaluation generalizes PU evaluation). If $\Sigma, \Theta, e \Downarrow_{p c} \Sigma^{\prime}, V$, then $\Sigma, \Theta, e \Downarrow_{p c} \Sigma^{\prime}, V$.

Proof. In the auxiliary material for this paper.

Again, the converse to this theorem does not hold, since Figure 1 shows an execution that gets stuck under the permissive upgrade semantics but not under the faceted semantics.

\section{Facet Declassification}

For many real systems, non-interference is too strong of a restriction. Often a certain amount of information leakage is acceptable, and even desirable. Password checking is the canonical example; while one bit of information about the password may leak, the system may still be deemed secure. Declassification is this process of making confidential data public in a controlled manner.

\footnotetext{
${ }^{3}$ In [6], these partially leaked values were represented as $R^{P}$, with a superscript $P$ denoting partially leaked.
}

In the context of multi-process execution [13], declassification is rather challenging. The $L$ and $H$ processes must be coordinated in a careful manner, with all of the attendant problems involved in sharing data between multiple processes. Additionally, allowing declassification may re-introduce timing channels and the termination channel, losing major benefits of the multi-execution approach. In contrast, faceted evaluation makes declassification fairly straightforward. The public and confidential facets are tied together in a single faceted value during execution, so declassification simply requires restructuring the faceted value to migrate information from one facet to another.

Providing a declassification operation with no restrictions invalidates most security guarantees. For instance, an attacker could declassify a user's password, or overwrite data that would be declassified later by legitimate code. In this manner, valid code intending to declassify the result of a password check might instead be duped into declassifying the password itself.

To provide more reliable security guarantees in the presence of declassification with faceted values, we show how to perform robust declassification [40], which guarantees that an active attacker, able to introduce code, is no more powerful than a passive attacker, who can only observe the results. (We use robust declassification as an illustrative example, but faceted values could also support other approaches to declassification.)

Robust declassification depends on a notion of integrity, which in turn requires that we distinguish between the terms label and principal. In particular, we introduce a separate notion of principals $(P)$ into our formalism. A label $k$ then marks data as being secret $\left(\mathrm{S}^{P}\right)$ or as being low-integrity or untrusted $\left(\mathrm{U}^{P}\right)$, both from the perspective of a particular principal $P^{4}$.

$$
\begin{aligned}
& P \in \text { Principal } \\
& k \in \text { Label } \quad:=\mathrm{S}^{P} \quad \text { secret to } P \\
& \text { | } \mathrm{U}^{P} \quad \text { untrusted by } P
\end{aligned}
$$

In the context of a principal $P$, we now have four possible views or projections of a computation, ordered by the subset relation.

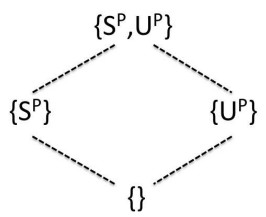

To help reason about multiple principals, we introduce the notation $L_{P}$ to abbreviate $L \cap\left\{\mathrm{S}^{P}, \mathrm{U}^{P}\right\}$, so that $L_{P}$ is one of the four views from the above combined confidentiality/integrity lattice. Note that in the absence of declassification, the projection theorem guarantees that each of these views of the computation are independent; there is no way for values produced in one view's computation to influence another view's computation.

We introduce an additional expression form declassify $y_{P} e$ for declassifying values with respect to a principal $P$. The rule [F-DECLASSIFY] in Figure 9 performs the appropriate robust declassification. Declassification cannot be performed by arbitrary unauthorized code, or else attackers could declassify all confidential data. Moreover, it is insufficient to allow code "owned" by $P$ to perform declassification, since attackers could leverage that code to declassify data on their behalf. Hence, the rule [F-DECLASSIFY] checks that the control path to this declassification operation has not been influenced by untrusted data, via the check $\mathrm{U}^{P} \notin p c$.

Robust declassification allows data to move from the $\left\{\mathrm{S}^{P}\right\}$ view to the \{\} view, but never from the $\left\{\mathrm{S}^{P}, \mathrm{U}^{P}\right\}$ view to the $\left\{\mathrm{U}^{P}\right\}$ view.

\footnotetext{
${ }^{4}$ This security lattice could be further refined to indicate which other principal was distrusted by $P$, which would permit more fine-grained decisions.
} 
That is, secret data can be declassified only if it is trusted. The downgrade $_{P}$ function shown in Figure 9 performs the appropriate manipulation to declassify values. The following lemma clarifies that this function migrates values from the trusted secret view $\left\{\mathrm{S}^{P}\right\}$ to the trusted public view \{\} , but not into any other view.

Lemma 5. For any value $V$ and view $L$ :

$L\left(\right.$ downgrade $\left._{P}(V)\right)=\left\{\begin{array}{l}L(V) \text { if } L_{P} \neq\{\} \\ L^{\prime}(V) \text { if } L_{P}=\{\}, \text { where } L^{\prime}=L \cup\left\{\mathrm{S}^{P}\right\}\end{array}\right.$

Proof. In the auxiliary material for this paper.

In the presence of declassification, the projection theorem does not hold for the public trusted view \{\} since that view's computation may be influenced by declassified data. However, the projection theorem still holds for other views. To prove this relaxed version of the projection theorem, we extend the standard semantics to treat declassification as the identity operation:

$$
\begin{aligned}
& \text { [S-DECLASSIFY] } \\
& \frac{\sigma, \theta, e \downarrow \sigma^{\prime}, V}{\sigma, \theta, \text { declassify }_{P} e \downarrow \sigma^{\prime}, V}
\end{aligned}
$$

Theorem 5 (Projection Theorem with Declassification). Suppose

$$
\Sigma, \Theta, e \Downarrow_{p c} \Sigma^{\prime}, V
$$

For any view $L$ for which $p c$ is visible, and where $L_{P} \neq\{\}$ for each $P$ used in a declassification operation, we have:

$$
L(\Sigma), L(\Theta), L(e) \downarrow L\left(\Sigma^{\prime}\right), L(V)
$$

Proof. In the auxiliary material for this paper.

As a result, non-interference also holds for these same views.

Theorem 6 (Termination Insensitive Non-Interference with Declassification). Suppose $L_{P} \neq\{\}$ for each $P$ used in a declassification operation and

Then:

$$
\begin{array}{ll}
\Sigma_{1} \sim_{L} \Sigma_{2} & \Sigma_{1}, \Theta_{1}, e \Downarrow_{\emptyset} \Sigma_{1}^{\prime}, V_{1} \\
\Theta_{1} \sim_{L} \Theta_{2} & \Sigma_{2}, \Theta_{2}, e \Downarrow_{\emptyset} \Sigma_{2}^{\prime}, V_{2}
\end{array}
$$

$$
\Sigma_{1}^{\prime} \sim_{L} \Sigma_{2}^{\prime} \quad V_{1} \sim_{L} V_{2}
$$

Proof. Follows from Theorem 5 via a proof similar to Theorem 2.

\section{JavaScript Implementation in Firefox}

We incorporate our ideas for faceted evaluation into Firefox through the Narcissus [15] JavaScript engine and the Zaphod [26] Firefox plugin. The ZaphodFacets implementation [4] extends the faceted semantics to handle the additional complexities of JavaScript. Exceptions are particularly tricky, and we halt execution if an exception may leak information.

We added two new primitives to the language. The makePrivate function turns a value into a faceted value with a public facet of undefined ${ }^{5}$. This approach allows developers to specify a different public value through the JavaScript idiom for specifying default values. The following code sets $\mathrm{x}$ to a faceted value of $\langle k ? 42: 0\rangle$. The high value of $x$ is set to 42 , since $(42 \| \mid 0)===42$; the low value will be 0 , since (undefined $\| \mid 0$ ) $===0$.

$\operatorname{var} \mathrm{x}=$ makePrivate (42) $\| \mathbf{|} 0$;

The second primitive is a getPublic function that extracts the public value of its input. For example, with the above code defining $\mathrm{x}$, getPublic (x) would return 0 . Generally, the browser's security

\footnotetext{
${ }^{5}$ A string specifying the principal can be given as the second argument if multiple principals are required.
}

policy should use these two functions (or variants) on all input/output boundaries of the system in order to appropriately label data as it comes in and to appropriately monitor data as it goes out.

To track information flow through the Document Object Model (DOM), our implementation uses the dom.js DOM implementation written in JavaScript [17], to preventing the attacker from sanitizing data by writing it to the DOM and later rereading it. Our implementation is available online with some examples [4], including the code from Figure 1.

\subsection{Cross-Site Scripting (XSS) Example}

To illustrate how our controls can be useful for enforcing practical defenses, we consider an example of a webpage with an XSS vulnerability. Our controls do not prevent XSS attacks. Rather, they provide an additional layer of defense, reducing an attack's power.

We specify a simple policy that the value of all password elements should be treated as confidential. Furthermore, any attempts to load files from a different origin should use the public facet; the server hosting the website, however, should see the true value.

In our example, the web developer is making use of a library for hashing passwords on the client side. The library is benign, but an attacker uses an XSS vulnerability in the page to wrap the hashing library and export the password to evil.com, a site under the attacker's control. The injected code is given below:

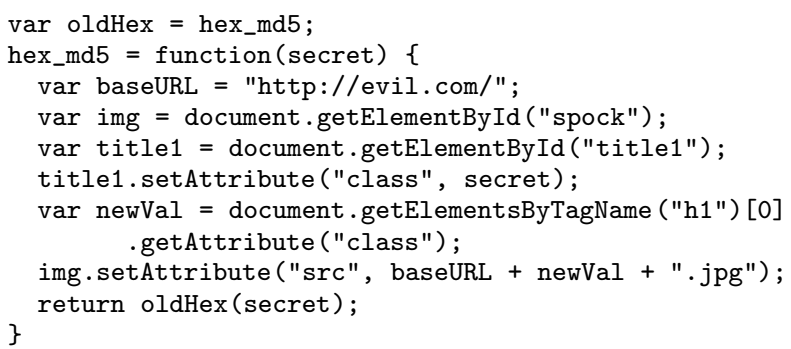

The attack attempts to leak the password by loading an image from evil.com, incorporating the password into the name of the requested image. However, in an attempt to evade our controls, it first writes the password to the class attribute of the title1 element and then rereads it from the first $\mathrm{h} 1$ element. Without knowledge of the DOM structure of the page, it is not possible to know whether this code leaks information. However, with dom.js we persist the different facets of secret to the DOM so that no security information is lost. While the page can only render a single facet, it is critical that we maintain other views of the document.

With this example, evil.com sees only the public facet of secret, not the true password. Trusted same-origin sources do see the true value, and therefore work correctly with the page.

While our example policy is far from complete, we use it to illustrate how our mechanism can enforce different information flow policies. A richer policy could specify a variety of fields and potential output channels. Furthermore, we imagine that browsers would wish to allow web developers to specify application-specific sensitive fields, such as credit card numbers, and allow users to protect information that they considered confidential (for instance, restricting the release of geolocation information).

\subsection{Performance Results}

Our approach is similar to Devriese and Piessens's work on secure multi-execution [13]. To understand the performance tradeoff between these two approaches we also implemented both sequential and concurrent versions of secure multi-execution in Narcissus, and compared their performance to faceted execution.

Our tests were performed on a MacBook Pro running OS X version 10.6.8. The machine had a $2.3 \mathrm{GHz}$ Intel Core 17 processor with 4 cores and $8 \mathrm{~GB}$ of memory. For our benchmark, we used 
Figure 10: Faceted Evaluation vs. Secure Multi-Execution

\begin{tabular}{c|r|r|r}
\multirow{2}{*}{ \# principals } & \multicolumn{3}{|c}{ Times in ms } \\
\cline { 2 - 4 } & \multicolumn{2}{|c|}{$\begin{array}{c}\text { Secure multi-execution } \\
\text { sequential }\end{array}$} & $\begin{array}{c}\text { Faceted } \\
\text { execution }\end{array}$ \\
\hline 0 & 273,774 & 283,450 & 310,561 \\
1 & 513,561 & 283,503 & 348,725 \\
2 & 961,357 & 332,303 & 387,121 \\
3 & $1,783,609$ & 597,595 & 421,566 \\
4 & $3,324,480$ & $1,093,951$ & 461,543 \\
5 & $*$ & $1,981,927$ & 503,364 \\
6 & $*$ & $*$ & 540,618 \\
7 & $*$ & $*$ & 575,100 \\
8 & $*$ & $*$ & 614,150 \\
\hline A result of "** indicates a test that ran for more than one hour.
\end{tabular}

the crypto-md5 test from the SunSpider [38] benchmark suite. We modified this program to include 8 hashing operations with some inputs marked as confidential. Our test cases involve 0 through 8 principals. In each case, every principal marks one element as private; additional hash inputs are public. For example, test 1 hashes 1 confidential input and 7 public inputs. Test 8 hashes 8 confidential inputs, each marked as confidential by a distinct principal, and has no public hash inputs. Our results are summarized in Figure 10.

Our results highlight the tradeoffs between the different approaches. The sequential variant of secure multi-execution had the most lightweight infrastructure of the three approaches, reflected in its good performance when there are 0 principals. However, it can neither take advantage of multiple processors nor avoid unnecessary work. As a result, once even a single principal was involved, it was the worst performer. The time required roughly doubles with each additional principal.

Concurrent secure multi-execution outperforms our faceted evaluation implementation when the number of principals is small. However, as the number of principals increases, faceted evaluation quickly becomes the more efficient approach, since under secure multi-execution the number of processes increases exponentially compared to the number of principals. With three principals, faceted evaluation outperforms concurrent secure multi-execution in our tests. Beyond this point execution time for concurrent secure multi-execution roughly doubles with each added principal, as the elements in the lattice now outnumber the available cores.

\section{Related Work}

A few publications have discussed performing multiple executions to guarantee security properties. Capizzi et al.'s shadow executions [9] develop an approach similar to faceted values for use in securing information for desktop applications; they run both a public and a private copy of the application. The public copy can communicate with the outside world, but has no access to private data. The private copy has access to all private information but does not transmit any information over the network. With this elegant solution, confidentiality is maintained. Devriese and Piessens [13] extend this idea to JavaScript code with their secure multi-execution strategy, using a high and a low process to protect confidentiality in a similar manner. Our approach is similar in spirit, though we avoid overhead when code does not depend on confidential data. Kashyap et al. [23] clarify some properties of secure multi-execution.

Our semantics are closely related to work by Pottier and Simonet [28]. While they prove non-interference statically for Core $M L$, their proof approach involves a Core $\mathrm{ML}^{2}$ language that has expression pairs and value pairs, analogous to our faceted expressions and faceted values. Our work departs from theirs in that we evaluate labeled expressions and values to dynamically guarantee non-interference, rather than using them to make static guarantees.

Kolbitsch et al. [25] use a similar technique in Rozzle, a JavaScript virtual machine for symbolic execution designed to detect malware. Rozzle uses multi-execution (not to be confused with secure multi-execution) to explore multiple paths in a single execution, similar to faceted evaluation. Their technique treats environment-specific data as symbolic, and explores both paths whenever a value branches on a symbolic value. The principal difference, besides the application, is that faceted values represent a lattice of different views of data, while Rozzle's symbolic heap values represent a range of possible values for different environments.

Other research has previously studied information-flow analysis for JavaScript. Vogt et al. [36] track information flow in Firefox to defend against XSS attacks. Russo and Sabelfeld [30] study timeout mechanisms. Russo et al. [32] discuss dynamic tree structures, with obvious applications to the DOM. Bohannon et al. [8] consider non-interference in JavaScript's reactive environment. Chugh et al. [11] create a framework for information flow analysis with "holes" for analyzing dynamically evaluated code. Dhawan and Ganapathy [14] discuss JavaScript-based browser extensions (JSEs). Jang et al. [21] give an excellent overview of how JavaScript is used to circumvent privacy defenses.

Information flow analysis largely traces its roots back to Denning [12]. Volpano et al. [37] codify Denning's approach as a type system, and also offer a proof of its soundness. Heintze and Riecke [19] design a type system for their purely functional SLam Calculus, which they extend to include mutable reference cells, concurrency, and integrity guarantees. Sabelfeld and Myers [33] offer an extensive survey of other research on information flow.

Myers [27] discusses JFlow, a variant of Java with security types to provide strong information flow guarantees. JFlow was the basis for Jif [22], a production-worthy language with information flow controls. Birgisson et al. [7] show how capabilities can guarantee information flow policies. Hunt and Sands [20] describe a flowsensitive type system. Russo and Sabelfeld [31] discuss the tradeoffs between static and dynamic analyses in some depth. Le Guernic et al. [18] examine code from branches not taken, increasing precision at the expense of run-time performance overhead. Shroff et al. [34] use a purely-dynamic analysis to track variable dependencies and reject more insecure programs over time.

Zdancewic [40] uses integrity labels to provide robust declassification. Askarov and Myers [2] consider checked endorsements. Chong and Myers [10] use a framework for application-specific declassification policies. Askarov and Sabelfeld [3] study a declassification framework specifying what and where data is released. Vaughan and Chong [35] infer declassification policies for Java programs. Askarov et al. [1] highlight complications of intermediary output channels. Rafnnson et al. [29] buffer output to reduce data lost from intermediary output channels and termination behavior. King et al. [24] study false alarms caused by implicit flows.

\section{Discussion}

Information flow non-interference is a tricky security property to enforce via dynamic monitoring, since it is a 2-safety property: non-interference can be refuted only by observing two executions (cmp. Theorem 2). Conversely, a 1-safety property can be refuted by observing a single execution, and so 1-safety properties are more amenable to dynamic enforcement. From this perspective, various prior techniques dynamically enforce a 1 -safety property that conservatively approximates the desired 2-safety property of non-interference, but this conservative approximation introduces false alarms on implicit flows. Interestingly, our projection property (Theorem 1) is a 1-safety property that suffices to prove noninterference (Theorem 2) without introducing false alarms. 
Acknowledgements We thank the anonymous POPL reviewers for their constructive feedback on this paper. We would also like to thank Brendan Eich, Andreas Gal, and Dave Herman for valuable discussions on information flow analysis, and David Flanagan and Donovan Preston for their help working with the dom.js project. This work was supported by NSF grant CNS-0905650.

\section{References}

[1] Aslan Askarov, Sebastian Hunt, Andrei Sabelfeld, and David Sands. Termination-insensitive noninterference leaks more than just a bit. In ESORICS '08, pages 333-348. Springer-Verlag, 2008.

[2] Aslan Askarov and Andrew Myers. A semantic framework for declassification and endorsement. In ESOP, pages 64-84, 2010.

[3] Aslan Askarov and Andrei Sabelfeld. Tight enforcement of information-release policies for dynamic languages. In IEEE Computer Security Foundations Symposium, pages 43-59, Washington, DC, USA, 2009. IEEE Computer Society.

[4] Thomas H. Austin. ZaphodFacetes github page. https://github. com/taustin/ZaphodFacets, 2011.

[5] Thomas H. Austin and Cormac Flanagan. Efficient purely-dynamic information flow analysis. In Workshop on Programming Languages and Analysis for Security, 2009.

[6] Thomas H. Austin and Cormac Flanagan. Permissive dynamic information flow analysis. In Proceedings of the 5th ACM SIGPLAN Workshop on Programming Languages and Analysis for Security, pages 1-12. ACM, 2010.

[7] Arnar Birgisson, Alejandro Russo, and Andrei Sabelfeld. Capabilities for information flow. In PLAS '11: Proceedings of the ACM SIGPLAN Fourth Workshop on Programming Languages and Analysis for Security. ACM, 2011.

[8] Aaron Bohannon, Benjamin C. Pierce, Vilhelm Sjöberg, Stephanie Weirich, and Steve Zdancewic. Reactive noninterference. In ACM Conference on Computer and Communications Security, pages 7990, 2009.

[9] R. Capizzi, A. Longo, V.N. Venkatakrishnan, and A.P. Sistla. Preventing information leaks through shadow executions. In ACSAC, pages $322-331$, dec 2008 .

[10] Stephen Chong and Andrew C. Myers. Security policies for downgrading. In CCS '04: Proceedings of the 11th ACM conference on Computer and communications security, pages 198-209, New York, NY, USA, 2004. ACM.

[11] Ravi Chugh, Jeffrey A. Meister, Ranjit Jhala, and Sorin Lerner. Staged information flow for JavaScript. In Conference on Programming Language Design and Implementation, 2009.

[12] Dorothy E. Denning. A lattice model of secure information flow. Communications of the ACM, 19(5):236-243, 1976.

[13] Dominique Devriese and Frank Piessens. Noninterference through secure multi-execution. Security and Privacy, IEEE Symposium on, 0:109-124, 2010.

[14] Mohan Dhawan and Vinod Ganapathy. Analyzing information flow in JavaScript-based browser extensions. In Annual Computer Security Applications Conference, 2009.

[15] Brendan Eich. Narcissus-JS implemented in JS, 2004. Available on the web at https://github. com/mozilla/narcissus/.

[16] J. S. Fenton. Memoryless subsystems. The Computer Journal, 17(2):143-147, 1974.

[17] Andreas Gal, David Flanagan, and Donovon Preston. dom.js github page. https://github.com/andreasgal/dom.js, accessed October 2011, 2011.

[18] Gurvan Le Guernic, Anindya Banerjee, Thomas P. Jensen, and David A. Schmidt. Automata-based confidentiality monitoring. In Asian Computing Science Conference on Secure Software, 2006.

[19] Nevin Heintze and Jon G. Riecke. The SLam calculus: Program- ming with secrecy and integrity. In Symposium on Principles of Programming Languages, 1998.

[20] Sebastian Hunt and David Sands. On flow-sensitive security types. In POPL, pages 79-90, 2006.

[21] Dongseok Jang, Ranjit Jhala, Sorin Lerner, and Hovav Shacham. An empirical study of privacy-violating information flows in javascript web applications. In ACM Conference on Computer and Communications Security, pages 270-283, 2010.

[22] Jif homepage, 2010. http://www.cs.cornell.edu/jif/, accessed October 2010.

[23] Vineeth Kashyap, Ben Wiedermann, and Ben Hardekopf. Timingand termination-sensitive secure information flow: Exploring a new approach. In IEEE Security and Privacy, 2011.

[24] Dave King, Boniface Hicks, Michael Hicks, and Trent Jaeger. Implicit flows: Can't live with 'em, can't live without 'em. In International Conference on Information Systems Security, pages 56-70, 2008.

[25] Clemens Kolbitsch, Benjamin Livshits, Benjamin Zorn, and Christian Seifert. Rozzle: De-cloaking internet malware. Technical Report MSR-TR-2011-94, Microsoft Research Technical Report, 2011.

[26] Mozilla labs: Zaphod add-on for the firefox browser, 2010. http: //mozillalabs.com/zaphod, accessed October 2010.

[27] Andrew C. Myers. JFlow: Practical mostly-static information flow control. In Symposium on Principles of Programming Languages, 1999.

[28] François Pottier and Vincent Simonet. Information flow inference for ML. Transactions on Programming Languages and Systems, 25(1):117-158, 2003.

[29] Willard Rafnsson and Andrei Sabelfeld. Limiting information leakage in event-based communication. In PLAS '11: Proceedings of the ACM SIGPLAN Fourth Workshop on Programming Languages and Analysis for Security. ACM, 2011.

[30] Alejandro Russo and Andrei Sabelfeld. Securing timeout instructions in web applications. In IEEE Computer Security Foundations Symposium, 2009.

[31] Alejandro Russo and Andrei Sabelfeld. Dynamic vs. static flowsensitive security analysis. In IEEE Computer Security Foundations Symposium. IEEE Computer Society, 2010.

[32] Alejandro Russo, Andrei Sabelfeld, and Andrey Chudnov. Tracking information flow in dynamic tree structures. In ESORICS, pages 86-103, 2009.

[33] Andrei Sabelfeld and Andrew C. Myers. Language-based information-flow security. Journal on Selected Areas in Coтmunications, 21(1):5-19, 2003.

[34] Paritosh Shroff, Scott F. Smith, and Mark Thober. Dynamic dependency monitoring to secure information flow. In Computer Security Foundations Symposium, 2007.

[35] Jeffrey Vaughan and Stephen Chong. Inference of expressive declassification policies. In IEEE Security and Privacy, 2011.

[36] Philipp Vogt, Florian Nentwich, Nenad Jovanovic, Engin Kirda, Christopher Krügel, and Giovanni Vigna. Cross-site scripting prevention with dynamic data tainting and static analysis. 2007.

[37] Dennis Volpano, Cynthia Irvine, and Geoffrey Smith. A sound type system for secure flow analysis. Journal of Computer Security, 4(2-3):167-187, 1996.

[38] Webkit.org. SunSpider JavaScript benchmark, 2011. http:// www . webkit .org/perf/sunspider/sunspider.html, accessed October 2011.

[39] Stephan Arthur Zdancewic. Programming languages for information security. PhD thesis, Cornell University, 2002.

[40] Steve Zdancewic. A type system for robust declassification. In 19th Mathematical Foundations of Programming Semantics Conference, 2003. 


\section{A. Proofs}

Lemma 4. Suppose pc is not visible to $L$ and that

$$
\Sigma, \Theta, e \Downarrow_{p c} \Sigma^{\prime}, V
$$

Then $L(\Sigma)=L\left(\Sigma^{\prime}\right)$.

Proof. We prove a stronger inductive hypothesis, namely that if $p c$ is not visible to $L$ and

1. $\Sigma, \Theta, e \Downarrow_{p c} \Sigma^{\prime}, V$ or

2. $\Sigma,\left(V_{1} V_{2}\right) \Downarrow_{p c} \Sigma^{\prime}, V$

then $L(\Sigma)=L\left(\Sigma^{\prime}\right)$.

The proof is by induction on the derivation of $\Sigma, \Theta, e \Downarrow_{p c} \Sigma^{\prime}, V$ and the derivation of $\Sigma,\left(V_{1} V_{2}\right) \Downarrow_{p c} \Sigma^{\prime}, V$, and by case analysis on the final rule used in that derivation.

- For cases [F-CONST], [F-VAR], [F-FUN], [F-BOT], [F-READ-IGNORE], and [FA-BOT], $\Sigma=\Sigma^{\prime}$. Therefore, $L(\Sigma)=L\left(\Sigma^{\prime}\right)$.

- Cases [F-DEREF], [F-APP], [F-LEFT], [F-RIGHT], [F-WRITE-IGNORE], [FA-FUN], [FA-LEFT], and [FA-RIGHT] hold by induction.

- For cases [F-SPLIT] and [FA-SPLIT], we note that since $p c$ is not visible to $L$, neither $p c \cup\{k\}$ nor $p c \cup\{\bar{k}\}$ are visible to $L$. Therefore these cases also hold by induction.

- For case [F-REF], $e=\operatorname{ref} e^{\prime}$. By the antecedents of this rule:

$$
\begin{gathered}
\Sigma, \Theta, e^{\prime} \Downarrow_{p c} \Sigma^{\prime \prime}, V^{\prime} \\
a \notin \operatorname{dom}\left(\Sigma^{\prime \prime}\right) \\
V^{\prime \prime}=\left\langle\left\langle p c ? V^{\prime}: \perp\right\rangle\right\rangle \\
\Sigma^{\prime}=\Sigma^{\prime \prime}\left[a:=V^{\prime \prime}\right]
\end{gathered}
$$

By induction, $L(\Sigma)=L\left(\Sigma^{\prime \prime}\right)$. Therefore, $\forall a^{\prime}$ where $a^{\prime} \neq a$, $L(\Sigma)\left(a^{\prime}\right)=L\left(\Sigma^{\prime}\right)\left(a^{\prime}\right)$. By Lemma $1, \Sigma^{\prime}(a)=\perp$. Since $a \notin \operatorname{dom}(\Sigma), \Sigma(a)=\perp$. Therefore $L(\Sigma)=L\left(\Sigma^{\prime}\right)$.

- For case [F-ASSIGN], $e=e_{a}:=e_{b}$. By the antecedents of the [F-ASSIGN] rule:

$$
\begin{gathered}
\Sigma, \Theta, e_{a} \Downarrow_{p c} \Sigma_{1}, V_{1} \\
\Sigma_{1}, \Theta, e_{b} \Downarrow_{p c} \Sigma_{2}, V \\
\Sigma^{\prime}=\operatorname{assign}\left(\Sigma_{2}, p c, V_{1}, V\right)
\end{gathered}
$$

By induction, $L(\Sigma)=L\left(\Sigma_{1}\right)=L\left(\Sigma_{2}\right)$. Therefore by Lemma 3, $L(\Sigma)=L\left(\Sigma^{\prime}\right)$.

- For case [F-READ], $e=\operatorname{read}(f)$. By the antecedents of this rule:

$$
\begin{gathered}
\Sigma(f)=v \cdot w \\
p c \text { visible to view }(f) \\
\Sigma^{\prime}=\Sigma[f:=w]
\end{gathered}
$$

Since $p c$ is not visible to $L, L \neq \operatorname{view}(f)$. Therefore, $L(\Sigma)(f)=L\left(\Sigma^{\prime}\right)(f)=\epsilon$.

- For case [F-WRITE], $e=$ write $\left(f, e^{\prime}\right)$. By the antecedents of this rule:

$$
\begin{gathered}
\Sigma, \Theta, e^{\prime} \Downarrow_{p c} \Sigma^{\prime \prime}, V \\
p c \text { visible to view }(f) \\
L^{\prime}=\text { view }(f) \\
v=L^{\prime}(V) \\
\Sigma^{\prime}=\Sigma^{\prime \prime}\left[f:=\Sigma^{\prime \prime}(f) \cdot v\right]
\end{gathered}
$$

By induction, $L\left(\Sigma^{\prime}\right)(f)=L\left(\Sigma^{\prime \prime}\right)(f)$. Since $p c$ is not visible to $L, L \neq L^{\prime}$. Therefore, $L(\Sigma)(f)=L\left(\Sigma^{\prime \prime}\right)(f)=L\left(\Sigma^{\prime}\right)(f)=$ $\epsilon$.
Theorem 1. Suppose

$$
\Sigma, \Theta, e \Downarrow_{p c} \Sigma^{\prime}, V
$$

Then for any view $L$ for which pc is visible,

$$
L(\Sigma), L(\Theta), L(e) \downarrow L\left(\Sigma^{\prime}\right), L(V)
$$

Proof. We prove a stronger inductive hypothesis, namely that for any view $L$ for which $p c$ is visible:

1. If $\Sigma, \Theta, e \Downarrow_{p c} \Sigma^{\prime}, V$ then $L(\Sigma), L(\Theta), L(e) \downarrow L\left(\Sigma^{\prime}\right), L(V)$.

2. If $\Sigma,\left(V_{1} V_{2}\right) \quad \downarrow_{p c} \quad \Sigma^{\prime}, V$ then $L(\Sigma), \theta\left[x:=L\left(V_{2}\right)\right], e^{\prime} \downarrow$ $L\left(\Sigma^{\prime}\right), L(V)$ where $L\left(V_{1}\right)=\left(\lambda x \cdot e^{\prime}, \theta\right)$.

The proof is by induction on the derivation of $\Sigma, \Theta, e \Downarrow_{p c} \Sigma^{\prime}, V$ and the derivation of $\Sigma,\left(V_{1} V_{2}\right) \Downarrow_{p c} \Sigma^{\prime}, V$, and by case analysis on the final rule used in that derivation.

- For case [F-CONST], $e=c$. Since $\Sigma, \Theta, c \quad \downarrow_{p c} \quad \Sigma, c$ and $L(\Sigma), L(\Theta), c \downarrow L(\Sigma), c$, this case holds.

- For case [F-VAR], $e=x$. This case then holds since $\Sigma, \Theta, x \downarrow$ $\downarrow_{p c} \Sigma, \Theta(x)$ and $L(\Sigma), L(\Theta), x \downarrow L(\Sigma), L(\Theta(x))$.

- For case [F-REF], $e=$ ref $e^{\prime}$. Then by the antecedents of the [F-REF] rule:

$$
\begin{gathered}
\Sigma, \Theta, e^{\prime} \Downarrow_{p c} \Sigma^{\prime \prime}, V^{\prime} \\
a \notin \operatorname{dom}\left(\Sigma^{\prime \prime}\right) \\
V^{\prime \prime}=\left\langle\left\langle p c ? V^{\prime}: \perp\right\rangle\right\rangle \\
\Sigma^{\prime}=\Sigma^{\prime \prime}\left[a:=V^{\prime \prime}\right] \\
V=a
\end{gathered}
$$

By induction, $L(\Sigma), L(\Theta), L\left(e^{\prime}\right) \downarrow L\left(\Sigma^{\prime \prime}\right), L\left(V^{\prime}\right)$. Since $a \notin \operatorname{dom}\left(\Sigma^{\prime \prime}\right), a \notin \operatorname{dom}\left(L\left(\Sigma^{\prime \prime}\right)\right)$. By Lemma $1, L\left(V^{\prime \prime}\right)=$ $L\left(V^{\prime}\right)$. Since $\Sigma^{\prime}=\Sigma^{\prime \prime}\left[a:=V^{\prime \prime}\right], L\left(\Sigma^{\prime}\right)=L(\Sigma)[a:=$ $\left.L\left(V^{\prime}\right)\right]$. Therefore, by the [S-REF] rule, $L(\Sigma), L(\Theta)$, ref $e^{\prime} \downarrow$ $L\left(\Sigma^{\prime}\right), L(V)$.

- For case [F-DEREF], $e=! e^{\prime}$. Then by the antecedents of the [F-DEREF] rule:

$$
\begin{gathered}
\Sigma, \Theta, e^{\prime} \Downarrow_{p c} \Sigma^{\prime}, V^{\prime} \\
V=\operatorname{deref}\left(\Sigma^{\prime}, V^{\prime}, p c\right)
\end{gathered}
$$

By induction, $L(\Sigma), L(\Theta), L\left(e^{\prime}\right) \downarrow L\left(\Sigma^{\prime}\right), L\left(V^{\prime}\right)$. Since $V^{\prime}$ must be an address, the bottom value, or a faceted value where all the nodes are addresses or the bottom value, it must be the case that $L\left(V^{\prime}\right)$ is an address or the bottom value.

- If $a=L\left(V^{\prime}\right)$, then by Lemma $2 L(V)=L\left(\Sigma^{\prime}\right)(a)$. Therefore, by the [S-DEREF] rule, $L(\Sigma), L(\Theta), L\left(! e^{\prime}\right) \downarrow$ $L\left(\Sigma^{\prime}\right), L(V)$.

- If $\perp=L\left(V^{\prime}\right)$, then by Lemma $2 L(V)=\perp$. Therefore, by the [S-DEREF] rule, $L(\Sigma), L(\Theta), L\left(! e^{\prime}\right) \downarrow L\left(\Sigma^{\prime}\right), L(V)$.

- For case [F-ASSIGN], $e=\left(e_{a}:=e_{b}\right)$. By the antecedents of the [F-ASSIGN] rule:

$$
\begin{gathered}
\Sigma, \Theta, e_{a} \Downarrow_{p c} \Sigma_{1}, V_{1} \\
\Sigma_{1}, \Theta, e_{b} \Downarrow_{p c} \Sigma_{2}, V \\
\Sigma^{\prime}=\operatorname{assign}\left(\Sigma_{2}, p c, V_{1}, V\right)
\end{gathered}
$$

By induction

$$
\begin{aligned}
& L(\Sigma), L(\Theta), L\left(e_{a}\right) \downarrow L\left(\Sigma_{1}\right), L\left(V_{1}\right) \\
& L\left(\Sigma_{1}\right), L(\Theta), L\left(e_{b}\right) \downarrow L\left(\Sigma_{2}\right), L(V)
\end{aligned}
$$

Since $V_{1}$ must either be an address or a faceted value where all the nodes are addresses, it must be the case that $L\left(V_{1}\right)$ is an address. Let $a=L\left(V_{1}\right)$. By Lemma 3, $\forall a^{\prime} \neq a, L\left(\Sigma^{\prime}\right)\left(a^{\prime}\right)=$ $L\left(\Sigma_{2}\right)\left(a^{\prime}\right)$. Also by Lemma $3, L\left(\Sigma^{\prime}\right)(a)=L(V)$. Therefore, by the [S-ASSIGN] rule, $L(\Sigma), L(\Theta), L\left(e_{a}:=e_{b}\right) \downarrow L\left(\Sigma^{\prime}\right), L(V)$. 
- For case [F-FUn], $e=\lambda x \cdot e^{\prime}$. Then $\Sigma, \Theta, \lambda x \cdot e^{\prime} \downarrow_{p c} \Sigma,\left(\lambda x . e^{\prime}, \Theta\right)$ and $L(\Sigma), L(\Theta), L\left(\lambda x . e^{\prime}\right) \downarrow L(\Sigma), L\left(\left(\lambda x . e^{\prime}, \Theta\right)\right)$. This case holds since $L\left(\left(\lambda x . e^{\prime}, \Theta\right)\right)=\left(\lambda x . L\left(e^{\prime}\right), L(\Theta)\right)$.

- For case [F-APP], $e=\left(e_{a} e_{b}\right)$. By the antecedents of the [F-APP] rule:

$$
\begin{gathered}
\Sigma, \Theta, e_{a} \Downarrow_{p c} \Sigma_{1}, V_{1} \\
\Sigma_{1}, \Theta, e_{b} \Downarrow_{p c} \Sigma_{2}, V_{2} \\
\Sigma_{2},\left(V_{1} V_{2}\right) \Downarrow_{p c} \Sigma^{\prime}, V
\end{gathered}
$$

By induction

$$
\begin{gathered}
L(\Sigma), L(\Theta), L\left(e_{a}\right) \downarrow L\left(\Sigma_{1}\right), L\left(V_{1}\right) \\
L\left(\Sigma_{1}\right), L(\Theta), L\left(e_{b}\right) \downarrow L\left(\Sigma_{2}\right), L\left(V_{2}\right)
\end{gathered}
$$

$V_{1}$ must be a function, the bottom value $(\perp)$, or a faceted value where all the nodes are functions or $\perp$.

- If $\left(\lambda x \cdot e^{\prime}, \theta\right)=L\left(V_{1}\right)$, then it holds by induction that $L\left(\Sigma_{2}\right), \theta\left[x:=L\left(V_{2}\right)\right], e^{\prime} \downarrow L\left(\Sigma^{\prime}\right), L(V)$. Therefore, by the [S-APP] rule, $L(\Sigma), L(\Theta), L\left(e_{a} e_{b}\right) \downarrow L\left(\Sigma^{\prime}\right), L(V)$.

- Otherwise, $\perp=L\left(V_{1}\right)$. By Lemma 4 and the [F-APP-BOT] rule, it follows that $L\left(\Sigma^{\prime}\right)=L\left(\Sigma_{2}\right)$ and $L(V)=\perp$. Therefore $L\left(\Sigma_{2}\right), L(\Theta), L\left(e_{a} e_{b}\right) \downarrow L\left(\Sigma^{\prime}\right), L(V)$ by the [S-APP-BOT] rule.

- For case [F-LEFT], $e=\left\langle k ? e_{a}: e_{b}\right\rangle$. By the antecedents of this rule

$$
\begin{gathered}
k \in p c \\
\Sigma, \Theta, e_{a} \Downarrow p c \Sigma^{\prime}, V
\end{gathered}
$$

Therefore $L\left(\left\langle k ? e_{a}: e_{b}\right\rangle\right)=L\left(e_{a}\right)$, and this case holds by induction.

- Case [F-RIGHT] holds by a similar argument as [F-LEFT].

- For case [F-SPLIT], $e=\left\langle k ? e_{a}: e_{b}\right\rangle$. By the antecedents of the [F-SPLIT] rule:

$$
\begin{gathered}
\Sigma, \Theta, e_{a} \Downarrow_{p c \cup\{k\}} \Sigma_{1}, V_{1} \\
\Sigma_{1}, \Theta, e_{b} \Downarrow_{p c \cup\{\bar{k}\}} \Sigma^{\prime}, V_{2} \\
V=\left\langle k ? V_{1}: V_{2}\right\rangle
\end{gathered}
$$

- Suppose $k \in L$. Then $p c \cup\{k\}$ is visible to $L$ and $\forall L$ where $L$ is consistent with, we know that $L(e)=$ $L\left(e_{a}\right)$ and $L\left(V_{1}\right)=L(V)$. By induction we know that $L(\Sigma), L(\Theta), L\left(e_{a}\right) \downarrow L\left(\Sigma_{1}\right), L(V)$. Therefore Lemma 4 implies $L\left(\Sigma_{1}\right)=L\left(\Sigma^{\prime}\right)$,

- Conversely suppose $k \notin L$. Then $p c \cup\{\bar{k}\}$ is visible to $L$ and $L(e)=L\left(e_{b}\right)$ and $L\left(V_{2}\right)=L(V)$. By Lemma 4 we know that $L(\Sigma)=L\left(\Sigma_{1}\right)$. Therefore, $L\left(\Sigma_{1}\right), L(\Theta), L\left(e_{b}\right) \downarrow L\left(\Sigma^{\prime}\right), L(V)$ by induction.

- For case [F-вот], $e=\perp$. Since $\Sigma, \Theta, \perp \downarrow_{p c} \quad \Sigma, \perp$ and $L(\Sigma), L(\Theta), \perp \downarrow L(\Sigma), \perp$, this case holds.

- For [F-READ], $e=\operatorname{read}(f)$. By the antecedents of this rule,

$$
\begin{gathered}
\Sigma(f)=v . w \\
L^{\prime}=\text { view }(f) \\
p c \text { visible to } L^{\prime} \\
p c^{\prime}=L^{\prime} \cup\left\{\bar{k} \mid k \notin L^{\prime}\right\} \\
\Sigma^{\prime}=\Sigma[f:=w] \\
V=\left\langle\left\langle p c^{\prime} ? v: \perp\right\rangle\right\rangle
\end{gathered}
$$

- If $L=\operatorname{view}(f)$, then $L(V)=v$. This case holds since $L(\Sigma), L(\Theta), \operatorname{read}(f) \downarrow L\left(\Sigma^{\prime}\right), v$.

- Otherwise, $L \neq$ view $(f)$. Therefore $L(\Sigma)=L\left(\Sigma^{\prime}\right)$ since $L(\Sigma(f))=\epsilon$. Also, $L(e)=\perp$ and $L(V)=\perp$. Therefore, this case holds since $L(\Sigma), L(\Theta), \perp \downarrow L(\Sigma), \perp$.

- For [F-READ-IGNORE], $e=\operatorname{read}(f)$. By the antecedent of this rule, $p c$ not visible to view $(f)$. Therefore, $L(e)=\perp$. Since $\Sigma, \Theta, \operatorname{read}(f) \Downarrow_{p c} \Sigma, \perp$ and $L(\Sigma), L(\Theta), \perp \downarrow L(\Sigma), \perp$, this case holds.
- For [F-WRITE], $e=\operatorname{write}\left(f, e^{\prime}\right)$. By the antecedents of this rule,

$$
\begin{gathered}
\Sigma, \Theta, e^{\prime} \Downarrow_{p c} \Sigma^{\prime \prime}, V \\
p c \text { visible to view }(f) \\
L^{\prime}=\text { view }(f) \\
v=L^{\prime}(V) \\
\Sigma^{\prime}=\Sigma^{\prime \prime}\left[f:=\Sigma^{\prime \prime}(f) \cdot v\right]
\end{gathered}
$$

By induction, $L(\Sigma), L(\Theta), e^{\prime} \downarrow L\left(\Sigma^{\prime \prime}\right), L(V)$.

- If $L=L^{\prime}$, then $L(V)=v$. Since $L\left(\Sigma^{\prime}\right)=L\left(\Sigma^{\prime \prime}[f:=\right.$ $\left.\left.L\left(\Sigma^{\prime \prime}(f) \cdot v\right)\right]\right)$, it follows that $L(\Sigma), L(\Theta)$, write $\left(f, e^{\prime}\right) \downarrow$ $L\left(\Sigma^{\prime}\right), L(V)$.

- Otherwise, $L \neq L^{\prime}$. Therefore $L\left(\Sigma^{\prime}\right)=L\left(\Sigma^{\prime \prime}\right)$, since $L\left(\Sigma^{\prime \prime}(f)\right)=\epsilon$. Also, it must be the case that $L\left(\right.$ write $\left.\left(f, e^{\prime}\right)\right)=$ $e^{\prime}$. Therefore this case holds, since by induction $L(\Sigma), L(\Theta), e^{\prime} \downarrow$ $L\left(\Sigma^{\prime \prime}\right), L(V)$.

- For [F-WRite-IGNORE], $e=$ write $\left(f, e^{\prime}\right)$. By the antecedents of this rule,

$$
\begin{gathered}
\Sigma, \Theta, e^{\prime} \Downarrow_{p c} \Sigma^{\prime}, V \\
p c \text { not visible to view }(f)
\end{gathered}
$$

Therefore, $L(e)=L\left(e^{\prime}\right)$ By induction, $L(\Sigma), L(\Theta), e^{\prime} \downarrow$ $L\left(\Sigma^{\prime}\right), L(V)$.

- Both cases [FA-LEFT] and [FA-RIGHT] hold by induction.

- For case [FA-FUN], we have (by the antecedent of this rule) $\Sigma, \Theta\left[x:=V_{2}\right], e^{\prime} \Downarrow_{p c} \Sigma^{\prime}, V$. Therefore, it holds by induction that $L(\Sigma), L\left(\Theta\left[x:=L\left(V_{2}\right)\right]\right), L\left(e^{\prime}\right) \downarrow L\left(\Sigma^{\prime}\right), L(V)$.

- For case [FA-SPLIT], we know that $V_{1}=\left\langle k\right.$ ? $\left.V_{a}: V_{b}\right\rangle$. By the antecedents of the rule:

$$
\begin{gathered}
k \notin p c, \bar{k} \notin p c \\
\Sigma,\left(V_{a} V_{2}\right) \Downarrow_{p c \cup\{k\}} \Sigma_{1}, V_{a}^{\prime} \\
\Sigma_{1},\left(V_{b} V_{2}\right) \Downarrow p \cup\{\bar{k}\} \Sigma^{\prime}, V_{b}^{\prime}
\end{gathered}
$$

As in the [F-SPLIT] case, we consider two separate cases.

- Suppose $k \in L$. Then $p c \cup\{k\}$ is visible to $L$ and $L(V)=$ $L\left(V_{a}^{\prime}\right)$. Then $L(\Sigma), \theta\left[x:=L\left(V_{2}\right)\right], e^{\prime} \downarrow L\left(\Sigma_{1}\right), L(V)$ by induction where $L\left(V_{a}\right)=\left(\lambda x \cdot e^{\prime}, \theta\right)$. By Lemma 4 , $\mathrm{L}\left(\Sigma_{1}\right)=L\left(\Sigma^{\prime}\right)$.

- Suppose $k \notin L$. Then $p c \cup\{\bar{k}\}$ is visible to $L$ and $L(V)=L\left(V_{b}^{\prime}\right)$. By Lemma $4, L(\Sigma)=L\left(\Sigma_{1}\right)$. By induction, $L\left(\Sigma_{1}\right), \theta\left[x:=L\left(V_{2}\right)\right], e^{\prime} \downarrow L\left(\Sigma^{\prime}\right), L(V)$ where $L\left(V_{b}\right)=\left(\lambda x . e^{\prime}, \theta\right)$.

- For case [FA-вот], $V_{1}=\perp$. Since $L(\perp) \neq\left(\lambda x . e^{\prime}, \theta\right)$, this case vacuously holds.

Theorem 3. If $\Sigma, \Theta, e \Downarrow_{p c} \Sigma^{\prime}, V$ then $\Sigma, \Theta, e \Downarrow_{p c} \Sigma^{\prime}, V$.

Proof. The proof is by induction on the derivation of $\Sigma, \Theta, e \Downarrow_{p c}$ $\Sigma^{\prime}, V$ and by case analysis on the final rule used in that derivation.

- For case [NSU-CONST], $e=c$. This case then holds since $\Sigma, \Theta, c \Downarrow_{p c} \Sigma, c$ and $\Sigma, \Theta, c \Downarrow_{p c} \Sigma, c$.

- For case [NSU-VAR], $e=x$. This case then holds since $\Sigma, \Theta, x \Downarrow_{p c} \Sigma, \Theta(x)$ and $\Sigma, \Theta, x \Downarrow_{p c} \Sigma, \Theta(x)$.

- For case [NSU-FUN], $e=\lambda x \cdot e^{\prime}$. This case then holds since $\Sigma, \Theta, x \Downarrow_{p c} \Sigma,\left(\lambda x . e^{\prime}, \Theta\right)$ and $\Sigma, \Theta, x \Downarrow_{p c} \Sigma,\left(\lambda x . e^{\prime}, \Theta\right)$.

- For case [NSU-BOT], $e=\perp$. This case then holds since $\Sigma, \Theta, \perp \Downarrow_{p c} \Sigma, \perp$ and $\Sigma, \Theta, \perp \Downarrow_{p c} \Sigma, \perp$.

- case [NSU-APP]. Then $e=\left(e_{a} e_{b}\right)$. By the antecedents of this rule:

$$
\begin{gathered}
\Sigma, \Theta, e_{a} \Downarrow_{p c} \Sigma_{1},\left(\lambda x . e^{\prime}, \Theta^{\prime}\right) \\
\Sigma_{1}, \Theta, e_{b} \Downarrow_{p c} \Sigma_{2}, V^{\prime} \\
\Sigma_{2}, \Theta^{\prime}\left[x:=V^{\prime}\right], e^{\prime} \Downarrow_{p c} \Sigma^{\prime}, V
\end{gathered}
$$


By induction:

$$
\begin{gathered}
\Sigma, \Theta, e_{a} \Downarrow_{p c} \Sigma_{1},\left(\lambda x \cdot e^{\prime}, \Theta^{\prime}\right) \\
\Sigma_{1}, \Theta, e_{b} \Downarrow_{p c} \Sigma_{2}, V^{\prime}
\end{gathered}
$$

Therefore, by the [F-APP] rule it is sufficient to show that $\Sigma_{2},\left(\left(\lambda x . e^{\prime}, \Theta^{\prime}\right) V^{\prime}\right) \Downarrow_{p c} \Sigma^{\prime}, V$. Since $\Sigma_{2}, \Theta^{\prime}, e^{\prime} \Downarrow_{p c} \Sigma^{\prime}, V$ by induction, this case holds by the [FA-FUN] rule.

- case [NSU-APP-BOT]. Then $e=\left(e_{a} e_{b}\right)$. By the antecedents of this rule:

$$
\begin{gathered}
\Sigma, \Theta, e_{a} \Downarrow_{p c} \Sigma_{1}, \perp \\
\Sigma_{1}, \Theta, e_{b} \Downarrow_{p c} \Sigma^{\prime}, V^{\prime} \\
V=\perp
\end{gathered}
$$

By induction:

$$
\begin{gathered}
\Sigma, \Theta, e_{a} \Downarrow_{p c} \Sigma_{1}, \perp \\
\Sigma_{1}, \Theta, e_{b} \Downarrow_{p c} \Sigma^{\prime}, V^{\prime}
\end{gathered}
$$

Therefore, by the [F-APP] rule it is sufficient to show that $\Sigma^{\prime},\left(\perp V^{\prime}\right) \Downarrow_{p c} \Sigma^{\prime}, \perp$, which holds by the [FA-BOT] rule.

- case [NSU-APP-K]. Then $e=\left(e_{a} e_{b}\right)$. By the antecedents of this rule:

$$
\begin{gathered}
\Sigma, \Theta, e_{a} \Downarrow_{p c} \Sigma_{1},\left\langle k ?\left(\lambda x \cdot e^{\prime}, \Theta^{\prime}\right): \perp\right\rangle \\
\Sigma_{1}, \Theta, e_{b} \Downarrow_{p c} \Sigma_{2}, V^{\prime} \\
\Sigma_{2}, \Theta^{\prime}\left[x:=V^{\prime}\right], e^{\prime} \Downarrow_{p c}\{k\} \Sigma^{\prime}, V^{\prime \prime} \\
V=\langle k\rangle^{p c} V^{\prime \prime}
\end{gathered}
$$

By induction:

$$
\begin{gathered}
\Sigma, \Theta, e_{a} \Downarrow_{p c} \Sigma_{1},\left\langle k ?\left(\lambda x . e^{\prime}, \Theta^{\prime}\right): \perp\right\rangle \\
\Sigma_{1}, \Theta, e_{b} \Downarrow_{p c} \Sigma_{2}, V^{\prime}
\end{gathered}
$$

Therefore, by the [F-APP] rule it will suffice to show that $\Sigma_{2},\left(\left\langle k ?\left(\lambda x \cdot e^{\prime}, \Theta^{\prime}\right): \perp\right\rangle V^{\prime}\right) \Downarrow_{p c} \Sigma^{\prime}, V$.

- If $k \in p c$, then $\Sigma_{2},\left(\left\langle k ?\left(\lambda x . e^{\prime}, \Theta^{\prime}\right): \perp\right\rangle V^{\prime}\right) \Downarrow_{p c} \Sigma^{\prime \prime}, V^{\prime \prime}$ by the [FA-LEFT] rule. By the [FA-FUN] rule, $\Sigma_{2}, \Theta^{\prime}\left[x:=V^{\prime}\right], e^{\prime} \downarrow$ $\downarrow_{p c} \Sigma^{\prime \prime}, V^{\prime \prime}$. By induction, $\Sigma^{\prime \prime}=\Sigma^{\prime}$ and $V^{\prime}=V^{\prime \prime}$. Since $V=\langle k\rangle^{p c}\left\langle k ? V^{\prime \prime}: \perp\right\rangle=V^{\prime \prime}$, it holds that $V^{\prime \prime}=V$.

- Otherwise, by the [FA-SPLIT] rule:

$$
\begin{gathered}
\Sigma_{2},\left(\left(\lambda x . e^{\prime}, \Theta^{\prime}\right) V^{\prime}\right) \Downarrow_{p c} \Sigma_{3}, V_{3} \\
\Sigma_{3},\left(\perp V^{\prime}\right) \Downarrow_{p c} \Sigma_{4}, V_{4} \\
V_{5}=\left\langle\left\langle k ? V_{3}: V_{4}\right\rangle\right\rangle
\end{gathered}
$$

By induction, $\Sigma_{3}=\Sigma^{\prime}$ and $V_{3}=V^{\prime \prime}$. By the [FA-BOT] rule, $\Sigma_{4}=\Sigma^{\prime}$ and $V_{4}=\perp$. Therefore, $V_{5}=\left\langle\left\langle k ? V^{\prime \prime}: \perp\right\rangle\right\rangle=$ $\langle k\rangle^{p c} V^{\prime \prime}=V$.

- case [NSU-LABEL]. Then $e=\left\langle k ? e^{\prime}: \perp\right\rangle$. By the antecedent of this rule:

$$
\begin{gathered}
\Sigma, \Theta, e^{\prime} \Downarrow_{k \cup\{p c\}} \Sigma^{\prime}, V^{\prime} \\
V=\langle k\rangle^{p c} V^{\prime}
\end{gathered}
$$

By induction, $\Sigma, \Theta, e^{\prime} \Downarrow_{p c \cup\{k\}} \Sigma^{\prime}, V^{\prime}$.

- If $k \in p c$, then $p c \cup\{k\}=p c$ and $V=V^{\prime}$. Therefore, by the [F-LEFT] rule, $\Sigma, \Theta,\left\langle k ? e^{\prime}: \perp\right\rangle \Downarrow_{p c} \Sigma^{\prime}, V$.

- Otherwise, $k \notin p c$ and $\bar{k} \notin p c$. Therefore $V=\left\langle k\right.$ ? $V^{\prime}$ : $\perp\rangle$. By the [F-вот] rule, $\Sigma^{\prime}, \Theta, \perp \Downarrow_{p c \cup\{\bar{k}\}} \Sigma^{\prime}, \perp$. Therefore, $\Sigma, \Theta,\left\langle k ? e^{\prime}: \perp\right\rangle \downarrow_{p c \cup\{k\}} \Sigma^{\prime}, V$ by the [F-SPLIT] rule.

- case [NSU-REF]. Then $e=$ ref $e^{\prime}$. By the antecedents of this rule:

$$
\begin{gathered}
\Sigma, \Theta, e^{\prime} \Downarrow_{p c} \Sigma_{1}, V^{\prime} \\
a \notin \operatorname{dom}\left(\Sigma_{1}\right) \\
\Sigma^{\prime}=\Sigma_{1}\left[a:=\left\langle\left\langle p c ? V^{\prime}: \perp\right\rangle\right]\right.
\end{gathered}
$$

By induction, $\Sigma, \Theta, e^{\prime} \Downarrow_{p c} \Sigma_{1}, a$. Without loss of generality, we assume that both executions allocate the same address $a$. Therefore, $\Sigma, \Theta$, ref $e^{\prime} \Downarrow_{p c} \Sigma^{\prime}, a$ by the [F-REF] rule.

- Case [NSU-Deref]. Then $e=! e^{\prime}$. By the antecedents of this rule:

$$
\begin{gathered}
\Sigma, \Theta, e^{\prime} \Downarrow_{p c} \Sigma^{\prime}, V_{a} \\
V=\operatorname{deref}\left(\Sigma^{\prime}, a, p c\right)=\Sigma^{\prime}(a)
\end{gathered}
$$

By induction, $\Sigma, \Theta, e^{\prime} \Downarrow_{p c} \Sigma^{\prime}, a$. Therefore $\Sigma, \Theta, ! e^{\prime} \Downarrow_{p c}$ $\Sigma^{\prime}, V$ by the [DEREF] rule.

- case [NSU-ASSign]. Then $e=e_{a}:=e_{b}$. By the antecedents of this rule:

By induction:

$$
\begin{gathered}
\Sigma, \Theta, e_{a} \Downarrow_{p c} \Sigma_{1}, a \\
\Sigma_{1}, \Theta, e_{b} \Downarrow_{p c} \Sigma_{2}, V \\
p c=1 \mathrm{abel}\left(\Sigma_{2}(a)\right) \\
V^{\prime}=\langle\langle p c ? V: \perp\rangle \\
\Sigma^{\prime}=\Sigma_{2}\left[a:=V^{\prime}\right]
\end{gathered}
$$

$$
\begin{array}{ccc}
\Sigma, \Theta, e_{a} \Downarrow_{p c} & \Sigma_{1}, a \\
\Sigma_{1}, \Theta, e_{b} \Downarrow_{p c} & \Sigma_{2}, V
\end{array}
$$

- If $p c=\{\}$, then since $\operatorname{assign}\left(\Sigma_{2},\{\}, a, V\right)=\Sigma_{2}[a:=$ $V]$ it follows that $\Sigma, \Theta, e_{a}:=e_{b} \downarrow_{p c} \Sigma^{\prime}, V$ by the [F-ASSIGN] rule.

- Otherwise, $p c=\{k\}$ and $\Sigma_{2}(a)=\left\langle k ? V^{\prime \prime}: \perp\right\rangle$. Since $\operatorname{assign}\left(\Sigma_{2},\{k\}, a, V\right)=\Sigma_{2}\left[a:=V^{\prime}\right]$, it holds that $\Sigma, \Theta, e_{a}:=e_{b} \Downarrow_{p c} \Sigma^{\prime}, V$ by the [F-ASSIGN] rule.

- case [NSU-ASSIGN-BOT]. Then $e=e_{a}:=e_{b}$. By the antecedents of this rule:

$$
\begin{aligned}
& \Sigma, \Theta, e_{a} \Downarrow_{p c} \Sigma_{1}, \perp \\
& \Sigma_{1}, \Theta, e_{b} \Downarrow_{p c} \Sigma^{\prime}, V
\end{aligned}
$$

By induction:

$$
\begin{gathered}
\Sigma, \Theta, e_{a} \Downarrow_{p c} \Sigma_{1}, \perp \\
\Sigma_{1}, \Theta, e_{b} \Downarrow_{p c} \Sigma^{\prime}, V
\end{gathered}
$$

Since $\Sigma^{\prime}=\operatorname{assign}\left(\Sigma^{\prime}, p c, \perp, V\right)$, this case holds by the [F-ASSIGN] rule.

- case [NSU-ASSIGN-K]. Then $e=e_{a}:=e_{b}$. By the antecedents of this rule:

$$
\begin{gathered}
\Sigma, \Theta, e_{a} \Downarrow_{p c} \Sigma_{1},\langle k ? a: \perp\rangle \\
\Sigma_{1}, \Theta, e_{b} \Downarrow_{p c} \Sigma_{2}, V \\
p c \cup\{k\}=1 \text { abel }\left(\Sigma_{2}(a)\right) \\
V^{\prime}=\langle\langle p c \cup\{k\} ? V: \perp\rangle \\
\Sigma^{\prime}=\Sigma_{2}\left[a:=V^{\prime}\right]
\end{gathered}
$$

By induction:

$$
\begin{gathered}
\Sigma, \Theta, e_{a} \Downarrow_{p c} \Sigma_{1},\langle k ? a: \perp\rangle \\
\Sigma_{1}, \Theta, e_{b} \Downarrow_{p c} \Sigma_{2}, V
\end{gathered}
$$

Let $\Sigma^{\prime \prime}=\operatorname{assign}\left(\Sigma_{2}, p c,\langle k ? a: \perp\rangle, V\right)=\Sigma_{2}\left[a:=V^{\prime \prime}\right]$ where $V^{\prime \prime}=\left\langle\left\langle\{k\} ? V: \Sigma_{2}(a)\right\rangle\right\rangle$. Since it must be the case that $\Sigma_{2}(a)=\left\langle k ? V_{\text {old }}: \perp\right\rangle, V^{\prime \prime}=\langle k ? V: \perp\rangle$. Therefore, $\Sigma, \Theta, e_{a}:=e_{b} \Downarrow_{p c} \Sigma^{\prime}, V$ by the [F-ASSIGN] rule.

Theorem 4. If $\Sigma, \Theta, e \Downarrow_{p c} \Sigma^{\prime}, V$, then $\Sigma, \Theta, e \Downarrow_{p c} \Sigma^{\prime}, V$.

Proof. The proof is by induction on the derivation of $\Sigma, \Theta, e \Downarrow_{p c}$ $\Sigma^{\prime}, V$ and by case analysis on the final rule used in that derivation.

- Cases [NSU-CONST], [NSU-VAR], [NSU-FUn], [NSU-BOT], [NSU-APP], [NSU-APP-BOT], [NSU-APP-K], [NSU-LABEL], [NSU-REF], and [NSU-DEREF], hold by the same argument as in the proof for Theorem 3 .

- Case [PU-ASSIGN]. Then $e=e_{a}:=e_{b}$. By the antecedents of this rule:

By induction:

$$
\begin{gathered}
\Sigma, \Theta, e_{a} \Downarrow_{p c} \Sigma_{1}, a \\
\Sigma_{1}, \Theta, e_{b} \Downarrow_{p c} \Sigma_{2}, V \\
V^{\prime}=\left\langle\left\langle p c ? V: \Sigma_{2}(a)\right\rangle\right. \\
\Sigma^{\prime}=\Sigma_{2}\left[a:=V^{\prime}\right]
\end{gathered}
$$

$$
\begin{gathered}
\Sigma, \Theta, e_{a} \Downarrow_{p c} \Sigma_{1}, a \\
\Sigma_{1}, \Theta, e_{b} \Downarrow_{p c} \Sigma_{2}, V
\end{gathered}
$$

Since $\operatorname{assign}\left(\Sigma_{2}, p c, a, V\right)=\Sigma_{2}\left[a:=V^{\prime \prime}\right]$ where $V^{\prime \prime}=$ $\left\langle\left\langle p c ? V: \Sigma_{2}(a)\right\rangle\right\rangle=V^{\prime}$, it follows that $\Sigma, \Theta, e_{a}:=e_{b} \Downarrow p c$ $\Sigma^{\prime}, V$ by the [F-ASSIGN] rule. 
- case [PU-ASSIGN-K]. Then $e=e_{a}:=e_{b}$. By the antecedents of this rule:

$$
\begin{gathered}
\Sigma, \Theta, e_{a} \Downarrow_{p c} \Sigma_{1},\langle k ? a: \perp\rangle \\
\Sigma_{1}, \Theta, e_{b} \Downarrow_{p c} \Sigma_{2}, V \\
V^{\prime}=\left\langle\left\langle p c ? V: \operatorname{Sigma}_{2}(a)\right\rangle\right\rangle \\
\Sigma^{\prime}=\Sigma_{2}\left[a:=V^{\prime}\right]
\end{gathered}
$$

By induction:

$$
\begin{gathered}
\Sigma, \Theta, e_{a} \Downarrow_{p c} \Sigma_{1},\langle k ? a: \perp\rangle \\
\Sigma_{1}, \Theta, e_{b} \Downarrow_{p c} \Sigma_{2}, V
\end{gathered}
$$

Since $\operatorname{assign}\left(\Sigma_{2}, p c,\langle k ? a: \perp\rangle, V\right)=\Sigma_{2}\left[a:=V^{\prime \prime}\right]$ where $V^{\prime \prime}=\left\langle\left\langle p c ? V: \Sigma_{2}(a)\right\rangle\right.$, it follows that $\Sigma, \Theta, e_{a}:=e_{b} \Downarrow_{p c}$ $\Sigma^{\prime}, V$ by the [F-ASSIGN] rule.

Lemma 5. For any value $V$ and view $L$ :

$L\left(\right.$ downgrade $\left._{P}(V)\right)=\left\{\begin{array}{l}L(V) \text { if } L_{P} \neq\{\} \\ L^{\prime}(V) \text { if } L_{P}=\{\}, \text { where } L^{\prime}=L \cup\left\{\mathrm{S}^{P}\right\}\end{array}\right.$

Proof. The proof is by induction and case analysis on $V$.

- Case $V=r$ holds since downgrade ${ }_{P}(r)=r$.

- Case $V=\left\langle\mathrm{S}^{P}\right.$ ? $\left.V_{1}: V_{2}\right\rangle$. Let $V^{\prime}=$ downgrade $_{P}(V)=$ $\left\langle\mathrm{U}^{P} ?\left\langle\mathrm{~S}^{P} ? V_{1}: V_{2}\right\rangle: V_{1}\right\rangle$.

- If $\mathrm{S}^{P} \in L$, then $L(V)=L\left(V_{1}\right)=L\left(V^{\prime}\right)$.

- If $\mathrm{U}^{P} \in L$ and $\mathrm{S}^{P} \notin L$, then $L(V)=L\left(V_{2}\right)=L\left(V^{\prime}\right)$.

- Otherwise $\mathrm{U}^{P} \notin L$ and $\mathrm{S}^{P} \notin L$. Then $L^{\prime}(V)=L\left(V_{1}\right)=$ $L\left(V^{\prime}\right)$.

- Case $V=\left\langle\mathrm{U}^{P}\right.$ ? $\left.V_{1}: V_{2}\right\rangle$. Let $V^{\prime}=$ downgrade $_{P}(V)=$ $\left\langle\left\langle\mathrm{U}^{P}\right.\right.$ ? $V_{1}$ : downgrade $\left._{P}\left(V_{2}\right)\right\rangle$.

- If $\mathrm{U}^{P} \in L$, then $L(V)=L\left(V_{1}\right)=L\left(V^{\prime}\right)$

- Otherwise, $L(V)=L\left(V_{2}\right)$. Then this case holds by induction.

- Case $V=\left\langle l\right.$ ? $\left.V_{1}: V_{2}\right\rangle$ holds by induction.

\section{Theorem 5. Suppose}

$$
\Sigma, \Theta, e \Downarrow_{p c} \Sigma^{\prime}, V
$$

For any view $L$ for which $p c$ is visible, and where $L_{P} \neq\{\}$ for each $P$ used in a declassification operation, we have:

$$
L(\Sigma), L(\Theta), L(e) \downarrow L\left(\Sigma^{\prime}\right), L(V)
$$

Proof. The proof is by induction on the derivation of $\Sigma, \Theta, e \Downarrow p c$ $\Sigma^{\prime}, V$ and case analysis on the last rule used in that derivation.

- Cases [F-CONST], [F-VAR], [F-REF], [F-DEREF], [F-ASSIGN], [F-FUN], [F-APP], [F-LEFT], [F-RIGHT], [F-SPLIT], [F-READ], [F-READ-IGNORE], [F-WRITE], [F-WRITE-IGNORE], and [F-BOT] hold by a similar argument as in the proof for Theorem 1.

- For case [Declassify], $e=\operatorname{declassify~}_{P} e^{\prime}$. Then by the antecedents of this rule:

$$
\begin{gathered}
\Sigma, \Theta, e^{\prime} \Downarrow_{p c} \Sigma^{\prime}, V^{\prime} \\
\mathrm{U}^{P} \notin p c \\
V=\text { downgrade }_{P}\left(V^{\prime}\right)
\end{gathered}
$$

By induction:

$$
L(\Sigma), L(\Theta), L\left(e^{\prime}\right) \downarrow L\left(\Sigma^{\prime}\right), L\left(V^{\prime}\right)
$$

By Lemma 5, $L$ (downgrade $\left.{ }_{P}\left(V^{\prime}\right)\right)=L\left(V^{\prime}\right)=L(V)$. 